\title{
A closer look: High-resolution pore-scale simulations of solute transport and mixing through porous media columns
}

\section{Guillem Sole-Mari ( $\sim$ gsolemari@lbl.gov )}

Lawrence Berkeley National Laboratory https://orcid.org/0000-0002-9890-079X

\section{Diogo Bolster}

University of Notre Dame

Daniel Fernandez-Garcia

Universitat Politecnica de Catalunya

\section{Research Article}

Keywords: Mixing, Porous media, Column, Experiment, Computational Fluid Dynamics

Posted Date: June 22nd, 2021

DOI: https://doi.org/10.21203/rs.3.rs-625251/v1

License: (1) This work is licensed under a Creative Commons Attribution 4.0 International License.

Read Full License

Version of Record: A version of this preprint was published at Transport in Porous Media on January 6th, 2022. See the published version at https://doi.org/10.1007/s11242-021-01721-z. 
Transport in Porous Media manuscript No.

(will be inserted by the editor)

\title{
A closer look: High-resolution pore-scale simulations of solute transport and mixing through porous media columns
}

Guillem Sole-Mari $^{1, *} \cdot$ Diogo Bolster ${ }^{2}$ • Daniel Fernàndez-Garcia ${ }^{3}$

Received: date / Accepted: date

\begin{abstract}
Mixing is pivotal to conservative and reactive transport behaviors in porous media. Methods for investigating mixing processes include mathematical models, laboratory experiments and numerical simulations. The latter have been historically limited by the extreme computational resources needed for solving flow and transport at the microscopic scale within the complex pore structure of a three-dimensional porous medium, while dealing with a sufficiently large domain in order to generate meaningful emergent continuum-scale observables. We present the results of such a set of virtual column experiments, which have been conducted by taking advantage of modern High-Performance Computing infrastructure and Computational Fluid Dynamics software capable of massively parallel simulations. The computational approach has important advantages such as full control over the experimental conditions as well as high spatial and temporal resolution of measurements. We study the roles of Péclet number and grain size variability on emergent conservative and reactive transport behaviors. Hydrodynamic dispersion results agree with the empirical and theoretical literature and link dispersivity to median grain size, while elucidating the impact of grain-size variability on the critical Péclet number. Reactive transport results also indicate that the relative degree of incomplete mixing is related to the granular material's mean hydraulic radius, and not to the median grain size. When compared to a well-known laboratory experiment with similar configuration, less incomplete mixing is observed in our simulations. We offer a partial explanation for this discrepancy, by showing how an apparent non-linear absorbance-concentration relationship may induce laboratory measurement error in the presence of local concentration fluctuations.
\end{abstract}

Keywords Mixing $\cdot$ Porous media $\cdot$ Column $\cdot$ Experiment $\cdot$ Computational Fluid Dynamics

\section{Article Highlights}

- High-resolution numerical simulation experiments were conducted to study fluid-fluid mixing in porous (granular) media.

- Results unravel the roles of Péclet number and grain-size variability on emergent conservative and reactive transport.

- Non-linear light attenuation and local concentration fluctuations could cause measurement errors in physical experiments.

\footnotetext{
${ }^{1}$ Earth and Environmental Sciences Area, Lawrence Berkeley National Laboratory, Berkeley CA, United States

${ }^{2}$ Department of Civil and Environmental Engineering and Earth Sciences, University of Notre Dame, South Bend IN, United States

${ }^{3}$ Department of Civil and Environmental Engineering (DECA), Universitat Politècnica de Catalunya, Barcelona, Spain

* Corresponding author: Guillem Sole-Mari ·E-mail: gsolemari@lbl.gov
} 


\section{Introduction}

Mixing is a key element of flow and transport through porous media, playing a critical role in most aspects of solute plume behavior including homogenization, dilution, dispersion, and reaction. Accurately predicting mixing processes is critical for example to proper groundwater remediation design (Dybas et al, 2002), risk assessment (Bolster et al, 2009) and in-depth understanding of complex natural and engineered biogeochemical systems (Dentz et al, 2011) to name a few. Heterogeneities at multiple scales that result in complex flows, along with the nonlinear nature of mixing, make this challenging even for the case of a single-species conservative solute (Le Borgne et al, 2011). These challenges amplify when considering mixing-driven reactions, where multiple species can interact (Valocchi et al, 2019).

Recognizing the importance of the mixing process and the need for upscaled reactive transport models that implicitly account for it, numerous modeling approaches have been proposed in recent years to model mixing-limited reactive transport from a variety of perspectives (e.g., Oates, 2007; Benson and Meerschaert, 2008; Edery et al, 2009; Sanchez-Vila et al, 2010; Ding et al, 2013; Chiogna and Bellin, 2013; Hochstetler and Kitanidis, 2013; Paster et al, 2013, 2014; Porta et al, 2016; Schmidt et al, 2017, 2018, 2019; Ginn, 2018; Sole-Mari et al, 2020; Gurung and Ginn, 2020). In order to validate these new formulations, most authors test their models against laboratory experiments. But in many cases, high quality experimental data that enables this is not available; in others, their limitations are barely considered and the data are likely stretched beyond the purpose of original experimental design. This can have serious consequences on supposed and typically well-intended validation exercises.

A typical experimental setup to study mixing in porous media would consist of a column filled with a solid granular material and saturated with a fluid. At the beginning of the experiment, two perfectly segregated solutions contain the two reactants that generate a reaction product when in contact. One of the solutions resides in the column, and the other one is injected through the inlet. As it moves across the column, the initially flat interface that separates the two solutions will deform due to local velocity fluctuations, and both reactants will penetrate into their counterpart's territory much further than they would have done by molecular diffusion alone if the velocity field was perfectly uniform. In the non-reactive case, longitudinal hydrodynamic dispersion (spreading) can be reasonably well described by an effective Fick's law for sufficiently long timescales such that the fluid has traveled an average length equivalent to a few grain diameters. But this Fickian dispersion assumption fails at predicting the actual mixing (and therefore reaction), which is not directly driven by spreading but is instead limited by local pore-scale homogenization via diffusion.

Good-quality experimental datasets for the conditions described in the previous paragraph are rather scarce. Two by now famous laboratory experiments, namely those of Gramling et al (2002) and Raje and Kapoor (2000), have for years been used for testing and validating mathematical and numerical models of mixing-limited reactive transport. While these experimental data are tremendously valuable, they have some important limitations, regarding both (i) control over experimental conditions, and (ii) scale, frequency and accuracy of observations. Recent advances have allowed researchers to physically reproduce and measure pore-scale flows with high resolution (e.g., Souzy et al, 2020; Heyman et al, 2020). These notable works investigate hydrodynamic deformation and spreading within relatively small domains in the (virtual) absence of diffusion, but do not provide the full picture for the evolution of mixing and reactions.

Computational models, which are ubiquitous in hydrologic sciences, have to date, due to computational limitations, been somewhat incapable of reproducing with full pore-scale resolution the conditions of a typical column experiment like the one described above. Because of the minimum requirements of a reasonable domain size in both the transverse $\left(\approx 10^{2}\right.$ grain sizes, for statistical representativeness and in order to reduce the influence of lateral boundaries) and the longitudinal direction $(\approx 200$ grain sizes, in order to capture the evolution and regimes of the spreading and mixing processes), such a numerical experiment involves simulating flow and transport around tens of thousands of packed solid grains, which requires tremendous computational resources. With the emergence, over recent years, of High Performance Computing (HPC) available for academic research, including massively parallel supercomputers and numerical codes expressly built for working at such scales, this type of simulation has finally become feasible. 
As opposed to coarser continuum scales which typically involve a number of strong assumptions and homogenizing simplifications, the assumptions involved in a pore-scale simulation, where the full Navier-Stokes and Advection-Diffusion equations can be solved, are much less significant. Hence, the results can be seen as a nearexact replica of the equivalent physical process. Performing a virtual, pore-scale column experiment to investigate solute mixing can overcome some of the typical difficulties of physical models thanks to $(i)$ full control over the experimental setup and conditions, and (ii) high-resolution, accurate observations. This high degree of fidelity can then be used to establish precise links between the local and the upscaled transport features, and to study the role of various contributing factors with little uncertainty.

In this paper we present a new set of simulations of pore-scale flow and transport within columns filled randomly with solid grains and saturated with liquid. We examine solute transport behavior around an initially sharp interface separating two solutions as described previously in this Introduction. We explore a range of Péclet numbers and different degrees of grain size heterogeneity to study the impact of these two factors on both conservative and reactive transport observables. The paper is structured as follows. In $\S 2$, the computational methods are described in detail, including the generation of synthetic media (\$2.1) and the flow and transport simulations $(\S 2.2)$. In $\S 3$, the results are presented and analyzed from a continuum or coarse scale perspective, for both conservative $(\$ 3.1)$ and reactive transport (\$3.2). Finally, a summary and conclusions are reported in $\S 4$. See the Data availability statement at the end of this paper for a link to access the simulation data.

\section{Simulations}

In this Section we describe the simulation methods and the virtual setup, which is in many ways analogous to a physical column experiment designed to study hydrodynamic mixing in saturated porous media.

\subsection{Generation of synthetic porous media}

The synthetic media are built from spherical solid grains (emulating for example glass beads), packed randomly through simulated gravitational settling using the open-source software Blender, as proposed by Icardi et al (2014). The purpose of these settling simulations is to generate physically meaningful random porous media, while having full and exact knowledge of the pore-space geometry.

The grains used for the generation of the synthetic media have random diameters $d$ drawn from a log-normal distribution $p(d)$,

$$
p(d ; \mu, \sigma)=\frac{1}{d \sigma \sqrt{2 \pi}} \exp \left(-\frac{[\ln d-\mu]^{2}}{2 \sigma^{2}}\right) .
$$

This implies that the volume-weighted distribution (or mass-weighted distribution assuming constant solid density) is also log-normal,

$$
p_{\mathrm{w}}(d ; \mu, \sigma)=p\left(d ; \mu_{\mathrm{w}}, \sigma\right), \quad \mu_{\mathrm{w}}=\mu+3 \sigma^{2},
$$

which is a common and arguably fair assumption for granular materials (Gardner, 1956). Note that the volumeweighted or mass-weighted distribution (and not the frequency distribution (1)) is what one would usually infer from a typical granulometric analysis. All porous media are made comparable by fixing the mean hydraulic radius, $r$, defined by Carman (1937) as the ratio of the total resident fluid volume to the fluid-solid contact surface, which may be calculated from the grain-size distributions,

$$
r=\frac{n}{6(1-n)} \frac{\int d^{3} p(d) \mathrm{d} d}{\int d^{2} p(d) \mathrm{d} d}=\frac{n}{6(1-n)} \mathrm{e}^{\mu+\frac{5}{2} \sigma^{2}},
$$

where $n$ is the medium's porosity. For each of the different preset degrees of grain size variability $\sigma$, we set the mean $\log$ grain diameter $\mu$ as indicated by equation (4), such that the hydraulic radius is the same for all porous 

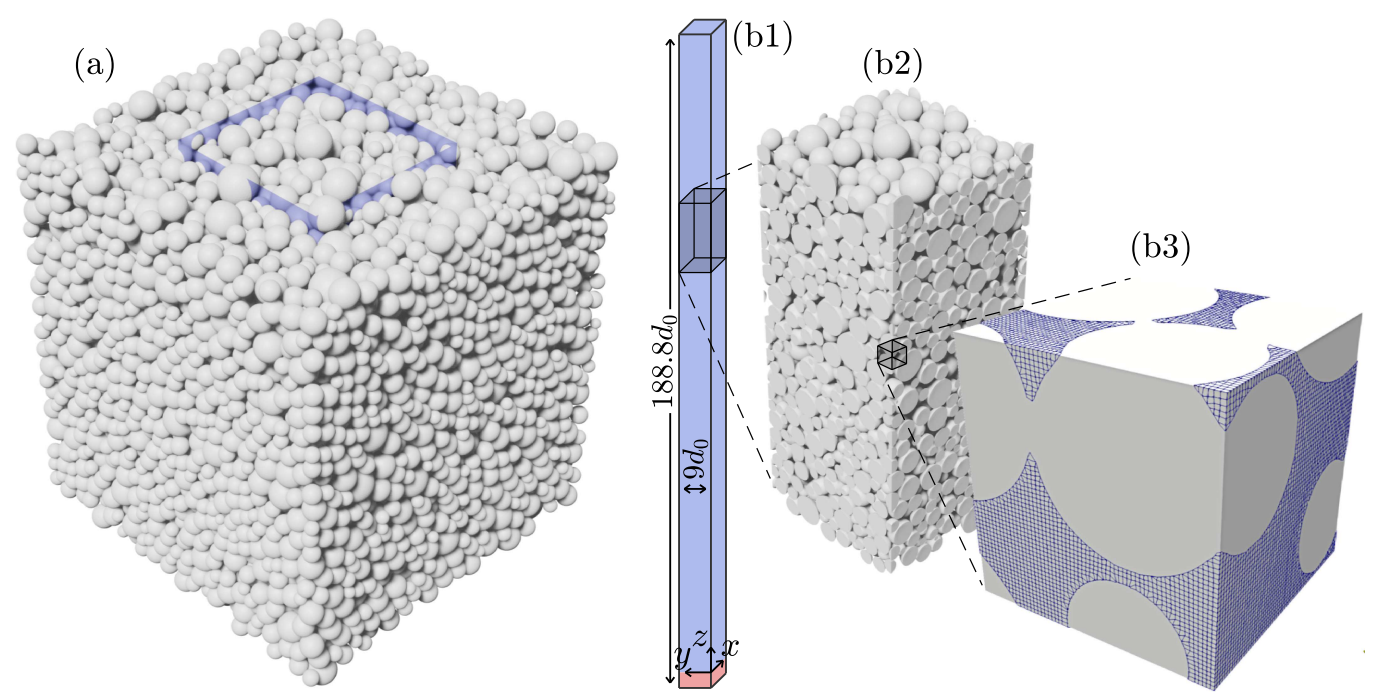

Fig. 1: (a) Example $\left(20 d_{0}\right)^{3}$ portion of the low-variance poly-disperse granular medium $(\sigma=0.25)$ resulting from the gravitational settling simulation, before removing the external region closer to the original walls (cutting planes depicted on top in blue); (b1) Full column dimensions and initial location of the concentration interface (at the coordinate axes origin) for all of our numerical experiments; (b2) Example portion ( $20 d_{0}$ in length, corresponding to the shaded region in (b1)) of the final low-variance poly-disperse granular medium $(\sigma=0.25)$; and (b3) Finite volume mesh used for the CFD simulations, depicted within an example $d_{0}^{3}$ region (size corresponding to the shaded region in (b2)).

media, i.e., $r=r_{0} \approx d_{0} / 10.6$, where the subscript 0 corresponds to the uniform case with $\sigma_{0}=0$ and grain diameter $d_{0}=\exp \left(\mu_{0}\right)$ :

$$
\mu=\mu_{0}-\frac{5}{2} \sigma^{2}+\ln \left(\frac{[1-n] n_{0}}{n\left[1-n_{0}\right]}\right) .
$$

The porosity $n$ is unknown a priori before each gravitational settling simulation. Nevertheless, we note that, far from the container boundaries (see below), $n$ only depends on the value of $\sigma$ (and not on $\mu$ ). Hence, $n$ is inferred by means of preliminary settling simulations with an arbitrary value for $\mu$, and then used to obtain the final $\mu$ from (3). By fixing the hydraulic radius $r$, according to the classical Kozeny-Carman theory (Kozeny, 1927; Carman, 1937), all media should have the same value of $k / n$ ( $k$ being permeability, dimensions $\mathrm{L}^{2}$ ), i.e., the same flux of fluid through a transverse cross-section per unit of pore-space surface given a fixed head gradient. This was later verified from the fluid flow simulation results, as detailed in \$2.2. We consider three different synthetic media with $\sigma=0, \sigma=0.25$ and $\sigma=0.50$, representing the mono-disperse, low-variance poly-disperse and moderate-variance poly-disperse case, respectively. In the latter, grain sizes span about one order of magnitude. Although granular materials' sizes can be even more heterogeneous, we were limited by computational and experimental setup constraints: excessively large grains would reduce the statistical representativeness of a single realization and increase boundary effects, whereas excessively small pores would not be discretized properly. For this same reason, the moderate-variance poly-disperse case is left-truncated so that $d / d_{0} \geq 0.2$. This truncation only excludes $0.5 \%$ of the grains and $0.003 \%$ of the grain volume in the distribution, and therefore its effect on the results is assumed to be negligible.

During a gravitational settling simulation, the randomly-sized grains are sequentially generated and placed at random positions above a square column-shaped container, which is composed of a horizontal base at the bottom and four lateral walls, with overall dimensions $20 d_{0} \times 20 d_{0} \times 235 d_{0}$. Grains keep falling and settling by gravity until the column is filled completely. The rigid-body physics parameter settings (such as friction and mass density) were fine-tuned until the porosity away from the boundary in the uniform grain-size case, $n=0.362$, was in close 

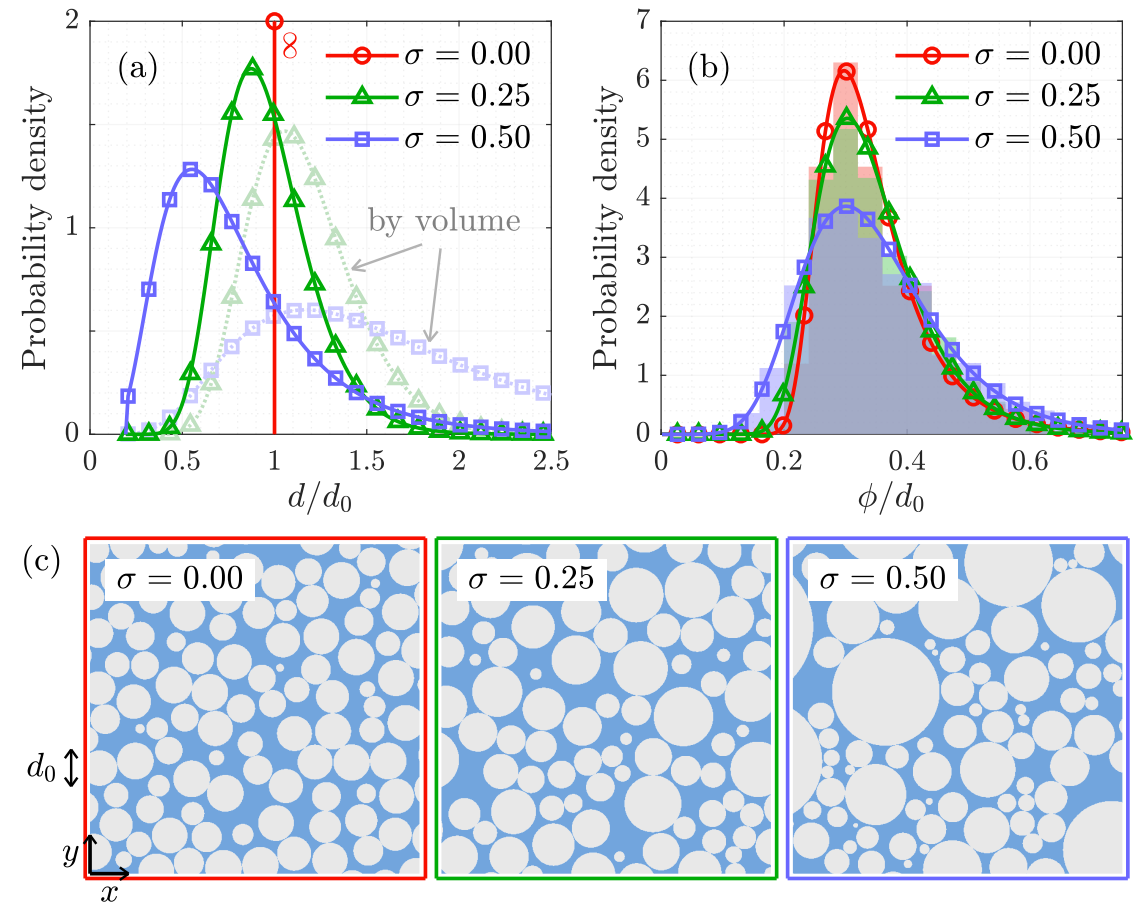

Fig. 2: (a) Grain-size distributions of the three generated media, by frequency (solid lines, equation (1)) and volume-weighted (clear dotted lines, equation (2)); (b) The inscribed pore-diameter frequency distributions for the three media (see the Appendix), as histograms (semitransparent bars) and fitted curves (solid lines); and (c)

Representative transverse cross-sections

agreement with the well-known random close-packed limit of $\approx 0.36$ (Scott and Kilgour, 1969; Finney and Bernal, 1970; Jaeger and Nagel, 1992). The same rigid-body physics parameter settings were then used for the low-variance and moderate-variance poly-disperse media, obtaining porosity values $n=0.343$ and $n=0.300$, respectively. Overlap errors at solid-solid contact points were measured by comparing the distance between centers of every two spherical grains in contact to the exact value which is the sum of the two respective radii. These overlap errors were deemed insignificant, being below $0.1 \%$ in $95 \%$ of the cases and never exceeding $0.5 \%$. Figure 1 (a) shows a cubic spatial subset of the settling simulation results for $\sigma=0.25$.

The structure of a solid body packing is strongly disrupted near rigid walls (Zou and Yu, 1995). In order to minimize the influence of boundaries on the flow and transport behavior, the final porous media are defined by extracting internal sub-regions from the settling simulation results. That is, out of an original granular medium generated in a settling simulation, with dimensions $20 d_{0} \times 20 d_{0} \times 235 d_{0}$, only an internal region with dimensions $9 d_{0} \times 9 d_{0} \times 188.8 d_{0}$ is kept, removing those areas closer to the original walls (see difference between Figures 1(a) and 1(b2)). By doing this, boundary effects are minimized and the measured behavior of statistical metrics such as the porosity within the final domains does not display any appreciable disruption near the final boundary. Each medium contains over 20,000 grains after clipping.

Figure 2(a) depicts the grain-size probability density functions of the three media. Respective representative cross-sections are shown in Figure 2(c). While all three media share the same mean hydraulic radius by construction, the volume-weighted median grain diameter $d_{50}$ increases (considerably) with increasing $\sigma$. These values are given in Table 1, along with other important configuration and characterization data. Quantities are indicated with representative dimensions of length and time (which correspond to a column length of 37.76 centimeters) for ease of interpretation, but all our analyses have been conducted in dimensionless form. In this work we use each medium's 
Table 1: Values of key metrics characterizing the structural and flow-related properties of the three generated granular media. Symbol $d_{50}$ is the volume-weighted median of $d$ (same for the median pore diameter $\phi_{50}$ ).

\begin{tabular}{l|ccccccccc}
\hline Medium name & $\begin{array}{c}\sigma \\
(-)\end{array}$ & $\begin{array}{c}\mu \\
(-)\end{array}$ & $\begin{array}{c}n \\
(-)\end{array}$ & $\begin{array}{c}r \\
(\mathrm{~mm})\end{array}$ & $\begin{array}{c}d_{50} \\
(\mathrm{~mm})\end{array}$ & $\begin{array}{c}\phi_{50} \\
(\mathrm{~mm})\end{array}$ & $\begin{array}{c}k \\
\left(\mathrm{~mm}^{2}\right)\end{array}$ & $\begin{array}{c}\tau \\
(-)\end{array}$ & $\begin{array}{c}u \\
(\mathrm{~mm} / \mathrm{s})\end{array}$ \\
\hline Mono-disperse & 0.00 & 0.693 & 0.362 & 0.189 & 2.000 & 0.799 & $2.755 \cdot 10^{-3}$ & 1.263 & 0.103 \\
Low-variance poly-disperse & 0.25 & 0.620 & 0.343 & 0.189 & 2.243 & 0.802 & $2.640 \cdot 10^{-3}$ & 1.269 & 0.103 \\
Moderate-variance poly-disperse & 0.50 & 0.349 & 0.300 & 0.189 & 3.000 & 0.912 & $2.252 \cdot 10^{-3}$ & 1.310 & 0.103 \\
\hline
\end{tabular}

$d_{50}$ as its default characteristic length. Inscribed pore diameter $(\phi)$ distributions by frequency are depicted in Figure 2(b) (see the Appendix for details on the evaluation of pore-size distributions), and the respective volume-weighted median pore diameters $\phi_{50}$ are given in Table 1 . We see that, while having markedly different median grain diameter values $d_{50}$, the three media with identical mean hydraulic radius $r$ also display nearly identical pore diameter mode in Figure 2(b), and similar values of the volume-weighted median pore diameter $\phi_{50}$.

\subsection{Flow and Transport}

The simulations were performed on the MareNostrum supercomputer (see Acknowledgments) using various components of the open-source Computational Fluid Dynamics (CFD) suite OpenFOAM. Simulations were performed in parallel on 3600 processors via domain decomposition, with a total computation time of 5 million core hours over a period of four months. The resulting dataset is over 6 TB in size (approximately $400 \mathrm{~GB}$ per individual simulation).

\subsubsection{Meshing}

Meshing of the pore space is performed in three steps. First, the spatial domain is discretized with a regular mesh of cubic cells of size $\Delta=d_{0} / 60$; this mesh size is selected through rigorous control of numerical error and mesh-independence of the simulation results, with particular attention to numerical diffusion in transport (see §2.2.3). This first step is performed using the utility blockMesh. Next, numerical cells occupied by the solid phase (spherical grains) are removed, and then the mesh is deformed so that the smooth geometry of the fluid-solid interface is faithfully represented (see Figure 1(b3)). These two last steps are calculated using the utility snappyHexMesh on 3600 processors in parallel.

\subsubsection{Navier-Stokes}

Once the domain mesh has been created, the next stage is to determine the steady-state solution of the incompressible Navier-Stokes (NS) equation which is assumed to dictate fluid flow within the pore space,

$$
(\mathbf{u} \cdot \nabla) \mathbf{u}-v \nabla^{2} \mathbf{u}=-\nabla h,
$$

where $\mathbf{u}(\mathbf{x})$ is the fluid velocity (dimensions $\left.\mathrm{LT}^{-1}\right), v$ is the kinematic viscosity of the fluid $\left(\mathrm{L}^{2} \mathrm{~T}^{-1}\right)$, and $h(\mathbf{x})$ is the hydraulic head expressed in units of kinematic pressure $\left(\mathrm{L}^{2} \mathrm{~T}^{-2}\right)$. Equation (5) is solved within the discretized pore space using the utility simpleFoam, which implements the SIMPLE algorithm.

A full-slip boundary condition is imposed on the lateral boundaries, so as to minimize their impact on the flow. Conversely, a classical no-slip condition is imposed at the contact between the fluid and the solid grains. Fixed hydraulic head is imposed at both ends of the column, generating a negative longitudinal mean head gradient $\langle\nabla h\rangle=[0,0,-g]^{\mathrm{T}}$ (brackets \langle\rangle stand for the entire-column average), where $g\left(\mathrm{LT}^{-2}\right)$ is different for each granular 
medium and is manually adjusted in order to obtain, for all cases, the same resulting mean fluid velocity over the entire column $\langle\mathbf{u}\rangle=[0,0, u]^{\mathrm{T}}$. The values of $u$ and $v$ are chosen such that the Reynolds number,

$$
\mathcal{R}=u d_{50} / v
$$

is equal to 0.2 in the mono-disperse case. This implies that flow is primarily controlled by viscous forces (term $\left.v \nabla^{2} \mathbf{u}\right)$ rather than inertial forces (term $\left.(\mathbf{u} \cdot \nabla) \mathbf{u}\right)$, which corresponds to the typical conditions of water flow through natural porous media.

Given the known values of the above-mentioned quantities, the saturated permeability $k$ of each porous material in the mean flow direction can be determined by

$$
k=v n u / g \text {. }
$$

The measured permeability values for all three media are given in Table 1 . In agreement with the assumptions involved in their design (see \$2.1), the three porous media display nearly identical values of $k / n$ (any discrepancies being of the order of $\pm 1 \%$ ). Furthermore, results agree very closely with the Kozeny-Carman equation (Carman, 1937; Bear, 1972), which predicts:

$$
k=n r^{2} / 5,
$$

where as mentioned in $\$ 2.1$, all three media have been designed to have identical expected values of the hydraulic radius $r$. The Kozeny-Carman equation (8) slightly under-predicts the measured permeability (7) in all three cases by about $6 \%$.

\subsubsection{Advection-Diffusion}

Once the stationary head and flow fields have been obtained, we simulate the transport of a conservative scalar $c(\mathbf{x}, t)$ (dimensions $\mathrm{ML}^{-3}$ ) which obeys the Advection-Diffusion Equation (ADE),

$$
\frac{\partial c}{\partial t}=-\mathbf{u} \cdot \nabla c+D \nabla^{2} c
$$

where $D\left(\mathrm{~L}^{2} \mathrm{~T}^{-1}\right)$ is the diffusion coefficient. The solution of (5), in the form of inter-cell fluxes, is provided as an input to solve (9) by means of the code scalarTransportFoam. A spectrum of different $D$ values is explored, such that the grain Péclet number,

$$
\mathcal{P}=u d_{50} / D
$$

ranges from $\mathcal{P}=10^{1}$ to $\mathcal{P}=10^{3.5}$ for the mono-disperse medium. We also perform the transport simulations in the poly-disperse media for a subset of these diffusion values, corresponding to the range $\mathcal{P}_{0}=10^{2}$ to $\mathcal{P}_{0}=10^{3}$, where $P_{0}=u d_{0} / D$. Accordingly, the Schmidt number,

$$
\mathcal{S}=v / D=\mathcal{P} / \mathcal{R}
$$

ranges from $\mathcal{S}=50$ to $\mathcal{S}>10^{4}$, implying that momentum diffusivity is much larger than mass diffusivity (as is typical for liquid solutions) in all tested scenarios.

A crucial aspect to control in high-Péclet number simulations is numerical diffusion. In order to minimize its impact on the results, we use a second-order TVD scheme with a van Leer flux limiter. We verified that numerical diffusion was negligible for the most restrictive grid Péclet number $\left(\mathcal{P}_{\mathrm{g}}=u \Delta / D \approx 50\right)$ by comparing constant-velocity transport simulations with the analytical solution. Instabilities were deemed negligible as well. Additionally, mesh refinement tests were performed in a small cubic subset of the granular domain with size $2 d_{0}$, again for the most restrictive case, verifying that transport results had indeed reached mesh-independence in the final configuration.

The initial condition for the transport simulations is $c(\mathbf{x}, 0)=c_{0} \theta(-z)$ (for $\mathbf{x}$ within the fluid phase), where $\theta$ is the Heaviside step function, emulating a sharp planar interface between two solutions at $z=0$ and $t=0$. The coordinate system is in fact defined relative to the initial position of the interface (see Figure 1(b1)), thus the inlet is 

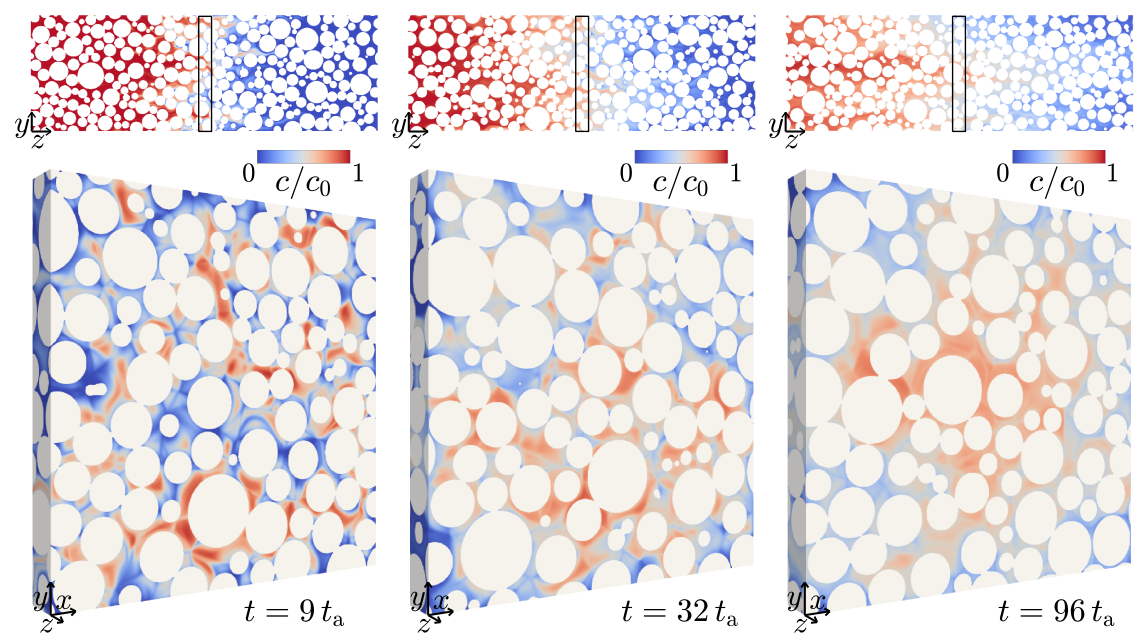

Fig. 3: Longitudinal cut view (top) and cross-section view at the moving interface (bottom) of pore-scale concentrations for conservative transport at three different times, with $\sigma=0.25$ and $\mathcal{P}=1155$. Mixing causes the amplitude of concentration fluctuations to decline over time.

located at $z_{\text {in }}=-4.5 d_{0}$. The solution with concentration $c_{0}$ is continuously entering the domain through the inlet, i.e., $c\left(\left[x, y, z_{\text {in }}\right]^{\mathrm{T}}, t\right)=c_{0}$ (in the fluid phase). Grain, lateral and outlet boundaries are assigned a zero-gradient condition. The total simulation time is $t_{\mathrm{end}}=250 d_{0} / u$, thus flushing the initial resident solution completely through the column of length $188.8 d_{0}$. Concentrations are recorded at $\Delta t=2.5 d_{0} / u$ time intervals.

Figure 3 shows the pore-scale concentrations in a region around the moving mixing front for $\sigma=0.25$ and $\mathcal{P} \approx 10^{3}$, through longitudinal cuts (top) and transverse slices (bottom), at three different times. Local concentration fluctuations, promoted by advective stretching of the initially sharp interface between the two solutions, gradually decrease due to diffusive mixing. Attentive examination of early-time concentrations reveals the presence of fluid stretching and folding patterns within pores (see Heyman et al (2020)).

\section{Macroscopic transport analysis}

Two scales of observation may be distinguished: The pore (or microscopic) scale, represented by quantities recorded in the numerical cells of the discretized pore space; and the coarse (or continuum) scale, represented by averaged quantities over some observation volume. These averaging volumes are defined as slabs of column with a longitudinal thickness equal to $d_{0}$ (see Figure 3 ). We indicate coarse-scale quantities with the overline notation,

$$
\bar{c}(z, t)=\frac{1}{V(z)} \int_{\Omega(z)} c(\mathbf{x}, t) \mathrm{d} \mathbf{x}
$$

where $\Omega(z)$ is an averaging volume as described above, centered at $[0,0, z]^{\mathrm{T}}$ and which includes only the fluid phase, and $V(z)=\int_{\Omega(z)} \mathrm{d} \mathbf{x}$.

In this Section the simulation results are analyzed on the basis of coarse-scale observations, like one would typically do in a physical column experiment. In $\$ 3.1$ we focus on longitudinal dispersion behavior, comparing to existing experimental and theoretical background. In $\$ 3.2$ we interpret the observations as pertaining to the reactive mixing between two solutions, and perform a qualitative comparison with the well-known benchmark physical experiment of Gramling et al (2002). 


\subsection{Conservative transport and dispersion}

In the particular transport problem addressed here, the longitudinal dispersion coefficient can be evaluated by interpreting $\bar{c}(z, t) / c_{0}$ as the complementary cumulative density function (ccdf) in $z$ of the transport of a unitary pulse injection $\bar{c}_{\delta}$, i.e.,

$$
\bar{c}_{\delta}(z, 0)=\left.\frac{1}{c_{0}} \frac{\partial c}{\partial z}\right|_{t=0}=\delta(z)
$$

where $\delta(z)$ is Dirac's delta function. As such, and as long as $\bar{c}\left(z_{\text {in }}, t\right)=1$ and $\bar{c}\left(z_{\text {out }}, t\right)=0$ (that is, the mixing front remains entirely within the column), the first spatial moment (center of mass) and the $i$ 'th centered spatial moments of $\bar{c}_{\delta}(z, t)$ can be computed from $\bar{c}(z, t)$ as

$$
m^{(1)}(t)=z_{\text {in }}+\frac{1}{c_{0}} \int_{z_{\text {in }}}^{z_{\text {out }}} \bar{c}(z, t) \mathrm{d} z, \quad m^{(n)}(t)=\left[z_{\text {in }}-m^{(1)}(t)\right]^{n}+\frac{i}{c_{0}} \int_{z_{\text {in }}}^{z_{\text {out }}}\left(z-m^{(1)}\right)^{i-1} \bar{c}(z, t) \mathrm{d} z,
$$

with integer $i \geq 2$. Then the longitudinal dispersion coefficient is defined as

$$
D_{\ell}(t)=\frac{1}{2} \frac{\partial m^{(2)}}{\partial t}
$$

\subsubsection{Asymptotic Dispersion}

Typically in porous media the dispersion coefficient reaches an asymptotic $(t \rightarrow \infty)$ value after a solute has traveled for some time within the same medium. Here we start by discussing the impact of experimental conditions on the asymptotic dispersion coefficient; how we identify the asymptote and extract the values from the data is explained in $\$ 3.1 .2$, where time-dependent behavior is analyzed.

One may relate the asymptotic hydrodynamic dispersion coefficient to the grain Péclet number and other micro-scale attributes. Such expressions can generally be written as (Bear, 1972)

$$
\frac{D_{\ell}(\infty)}{D}=\tau^{-2}+\alpha \mathcal{P} \beta(\mathcal{P})
$$

where $\alpha$ is a dimensionless dispersivity (generally assumed close to unity for a homogeneous granular material); $\beta(\mathcal{P}) \in[0,1]$ is a factor that quantifies the homogenizing impact that diffusion exerts on the variability of Lagrangian solute velocities, which usually satisfies $\beta(0)=0$ and $\beta(\infty)=1$; and $\tau>1$ is the tortuosity, which quantifies the typical arc-to-chord ratio within the medium in the longitudinal direction. The latter may be estimated from the fluid velocities as (Koponen et al, 1996)

$$
\tau=\frac{\int|\mathbf{u}|(\mathbf{x}) \mathrm{d} \mathbf{x}}{\int u_{z}(\mathbf{x}) \mathrm{d} \mathbf{x}},
$$

where $|\mathbf{u}|(\mathbf{x})$ and $u_{z}(\mathbf{x})$ are the magnitude and the longitudinal component of the fluid velocity, respectively. Although the advective tortuosity computed by (17) may differ slightly from the actual diffusive tortuosity, this is not expected to be critical for the high-Péclet conditions of this work. Note that the dimensionless dispersivity $\alpha$ employed here corresponds to normalizing the classical definition of dispersivity (which has units of length) by $d_{50}$.

As shown in Figure 4(a), the simulation results agree closely with (16) using the empirical expression for $\beta(\mathcal{P})$ found by Hiby (1959) for a packing of spherical glass-beads,

$$
\beta(\mathcal{P})=\frac{1}{1+\sqrt{\mathcal{P}_{\mathrm{c}} / \mathcal{P}}},
$$

where $\mathcal{P}_{\mathrm{c}}$ is a critical Péclet number. The best-fit parameter values (obtained by non-linear least squares regression analysis) in the mono-disperse medium are $\alpha=1.05$ and $\mathcal{P}_{\mathrm{c}}=228$. These are depicted in Figure 4(c) for all three media with their respective $95 \%$ confidence intervals. These results agree well with experimental works which report super-linear scaling of $D_{\ell}(\infty)$ with $\mathcal{P}$ for $1 \ll \mathcal{P}<400$ and linear behavior for $\mathcal{P}>400$ (Pfannkuch, 1963; Sahimi, 

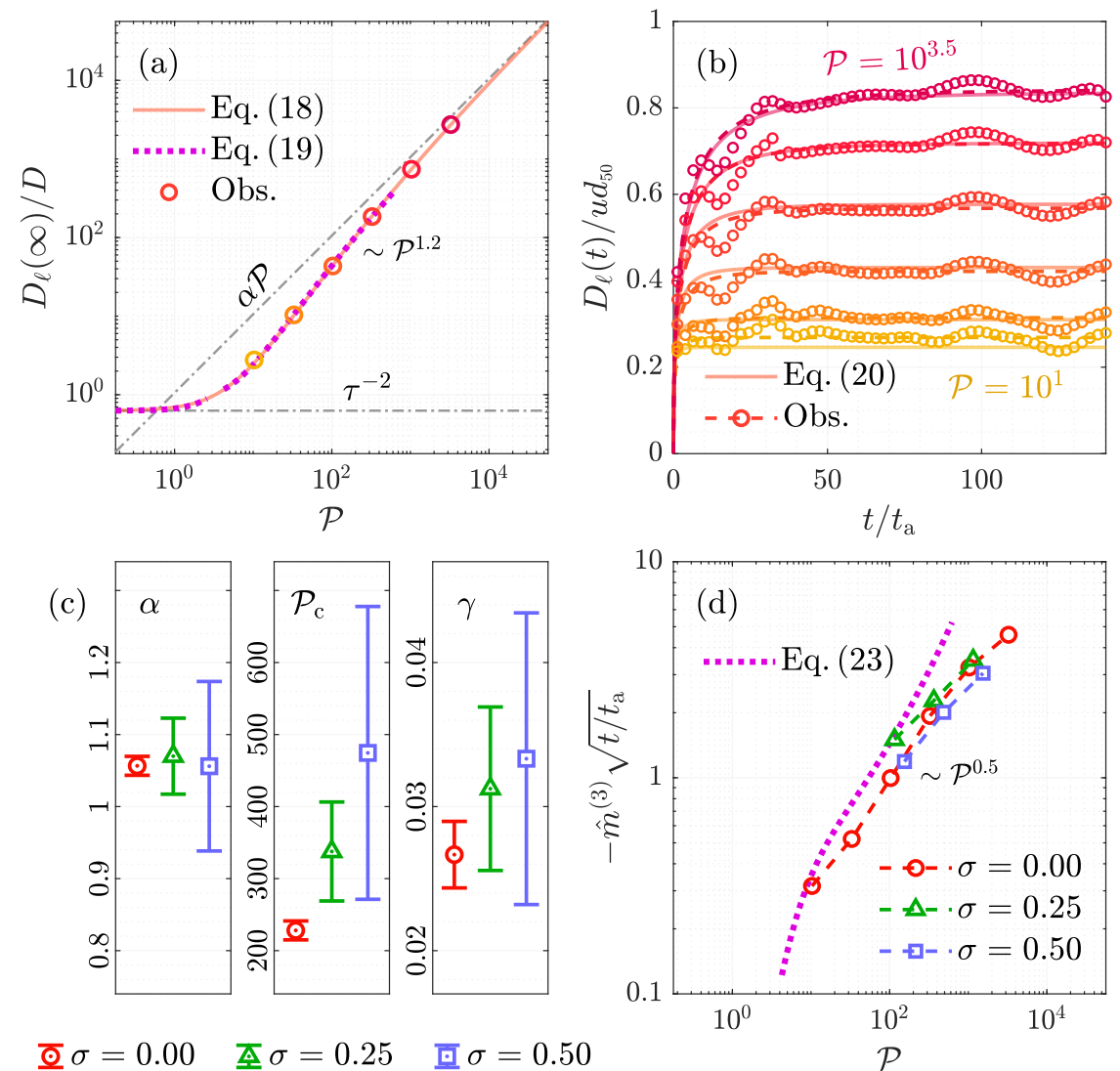

Fig. 4: (a) Observations (circles) of the asymptotic dispersion coefficient $D_{\ell}(\infty)$ as a function of the Péclet number $\mathcal{P}$ for the mono-disperse case $(\sigma=0)$, compared to two theoretical models (equations (16)-(19)), as well as the two limit regimes (gray dash-dotted lines) of equation (16) when $\mathcal{P} \ll 1$ and when $\mathcal{P} \gg \mathcal{P}_{\mathrm{c}}$; (b) Observations (circles) of the dispersion coefficient $D_{\ell}$ as a function of time for $\sigma=0$, fitted to complementary negative exponentials (dashed lines), compared to the model given by (17), (18), (20) and (21), with $\log \mathcal{P}$ ranging from 1 to 3.5 at 0.5 intervals; (c) $95 \%$ confidence intervals of the dimensionless model parameters $\alpha$ (dispersivity), $\mathcal{P}_{\mathrm{c}}$ (critical Péclet number) and $\gamma$ (critical time multiplier), for the three different degrees of grain-size variability given by $\sigma$; and (d) relative skewness, at late time $t=141.6 d_{0} / u$, of the longitudinal concentration profile in the simulations for the three different degrees of grain-size variability given by $\sigma$ (markers) compared to Saffman's model (equation (23)).

2011; Bijeljic et al, 2004, and references therein). The value $\alpha=1.05$ implies that the longitudinal dispersivity of the mono-disperse porous medium is equal to $\alpha d_{0}$, i.e., approximately equal to one grain diameter $d_{0}$. The asymptotic dispersion results relative to the diffusion coefficient, fitted against Péclet number to a single power law, would yield an effective scaling $\sim \mathcal{P}^{1.2}$, as indicated in Figure $4(\mathrm{a})$, in agreement with multiple experimental works (Bijeljic et al, 2004, and references therein). When $\mathcal{P} \gg \mathcal{P}_{\mathrm{c}}, \beta(\mathcal{P}) \approx 1$ and the longitudinal dispersion coefficient approaches $D_{\ell}(\infty) \approx \tau^{-2} D+\alpha d_{50} u$, which is the standard relationship between the dispersion coefficient and the mean fluid velocity used by many popular groundwater transport models and numerical codes. 
According to Saffman (1960), by idealizing the porous medium as a random network of capillaries of length $\lambda$, one would obtain the following expression for $\beta(\mathcal{P})$ :

$$
\beta(\mathcal{P})= \begin{cases}\frac{1}{15} \hat{\lambda} \mathcal{P}, & \text { for } \hat{\lambda} \mathcal{P} \ll 1, \\ \frac{1}{6} \ln \left(\frac{3}{2} \hat{\lambda} \mathcal{P}\right)-\frac{17}{72}-\frac{\psi^{2}}{48} \hat{\lambda} \mathcal{P}+\frac{4}{9 \hat{\lambda} \mathcal{P}}, & \text { for } 1 \ll \hat{\lambda} \mathcal{P} \ll 8 / \psi^{2},\end{cases}
$$

where $\hat{\lambda}=\lambda / d_{50}$ relates capillary length to the medium's characteristic distance, $\psi$ is the typical ratio between capillary radius and length, and $\alpha=\hat{\lambda}$ in (16). As shown in Figure 4(a), formulations (18) and (19) display close agreement within the applicability ranges of the latter, for $\lambda=0.78$ and $\psi=0.08$. The potential similarity between these two formulations was originally remarked by Saffman (1960). Note that, for $\mathcal{P} \ll 1$ (i.e., when pore-scale transport is diffusion-dominated), Saffman's $\beta$ scales as $\sim \mathcal{P}$ (i.e., $D_{\ell}(\infty) / D$ scales as $\sim \mathcal{P}^{2}$ ), similar to Taylor-Aris dispersion in a circular pipe (Aris and Taylor, 1956). Yet, in the intermediate regime $1 \ll \mathcal{P} \ll \mathcal{P}_{\mathrm{c}}$ both (18) and (19) exhibit non-quatradic scaling, which implies that Taylor-like dispersion does not emerge for that regime; this is in contrast with other models for $\beta(\mathcal{P})$ found in the literature (e.g., van Milligen and Bons, 2012), which propose that $D_{\ell}(\infty) \sim D \mathcal{P}^{2}$ for $1 \ll \mathcal{P} \ll \mathcal{P}_{\mathrm{c}}$. Such models do not agree as closely with the presented data.

Adopting Hiby's model, given by (16) and (18), the role of the grain-size variability (value of $\sigma$ ) on the upscaled transport parameters can be analyzed from Figure 4(c). The value of $\alpha$ stays consistently close to 1 for all three media, corroborating the prevalent assumption that dispersivity is approximately equal to the median grain diameter (see Pfannkuch, 1963; Bear, 1972). From a pore-size (instead of grain-size) perspective, the higher observed dispersivity $\alpha d_{50}$ in the poly-disperse cases may be linked to the increased pore-size variance (see Figure 2(b)) and consequent variability in flow velocities (e.g., Sahimi, 2011).

Also in Figure 4(c), we see that the value of the critical Péclet number $\mathcal{P}_{\mathrm{c}}$ is substantially higher in the polydisperse media $(\sigma>0)$ in comparison to the mono-disperse one $(\sigma=0)$, although this parameter's uncertainty is considerable due to the limited range of tested values of $\mathcal{P}$ and the more irregular behavior of $D_{\ell}(t)$ in the two poly-disperse media. In order to elucidate the impact of $\sigma$ on $\mathcal{P}_{\mathrm{c}}$, it is important to emphasize that this number marks the transition towards the pure fluid stretching-dominated regime in which diffusive mixing plays virtually no role in hydrodynamic dispersion. In other words, $\mathcal{P} \gg \mathcal{P}_{\mathrm{c}}$ represents the limiting case where transport is very strongly advection-dominated within most of the pores and thus a further increase in $\mathcal{P}$ has virtually no effect on the apparent dispersivity $D_{\ell} / u$ (i.e., $\beta(\mathcal{P}) \approx 1$ ). Following this reasoning one could anticipate a larger value of $\mathcal{P}_{\mathrm{c}}$ for larger values of $\sigma$, given the lower associated ratio of typical pore to grain size (Table 1), as well as the higher associated pore-size variability (Figure 2(b)); both these features would increase the critical $\mathcal{P}$ value (based on grain size) required for reaching widespread pore-scale "purely advective" conditions.

\subsubsection{Pre-asymptotic and non-Fickian behavior}

The temporal evolution towards the asymptotic dispersion regime is well fit to the complementary of a negative exponential,

$$
\frac{D_{\ell}(t)}{D}=\tau^{-2}+\alpha \mathcal{P} \beta(\mathcal{P})\left[1-\exp \left(-\sqrt{t / t_{\mathrm{c}}}\right)\right]
$$

where

$$
t_{\mathrm{c}}=\gamma \beta^{2}(\mathcal{P}) \mathcal{P}_{\mathrm{c}} t_{\mathrm{a}}
$$

is a critical time such that times $t \gg t_{\mathrm{c}}$ correspond to the asymptotic dispersion regime, $\gamma$ is a dimensionless parameter that depends on the medium, $\beta(\mathcal{P})$ is given by (18), and $t_{\mathrm{a}}=d_{50} / u$ is the characteristic advective time. Figure 4(b) shows the time-dependent dispersion coefficient data for the mono-disperse case with $\mathcal{P}$ ranging from $10^{1}$ to $10^{3.5}$, as well as the corresponding model fit (solid lines). Individual negative exponential curve fittings for each case are also depicted (dashed lines). The asymptotes of these individual best-fits are adopted as the experimental asymptotic dispersion coefficient values (see \$3.1.1 and Figure 4(a)).

It is worth noting from Figure 4(b) how the asymptotic (large $t / t_{\mathrm{a}}$ ) apparent dimensionless dispersivity $D_{\ell} / u d_{50}$ continues to increase noticeably with increasing $\mathcal{P}$ even for $\mathcal{P}>1000$. This implies that a single, Péclet-independent 
dispersivity value (i.e., $\beta=1$, which is a common assumption in transport models) may only be attained for extremely large $\mathcal{P}$; and at that point one might also have to consider the effects of non-laminar flow due to $\mathcal{R} \gg 1$.

The model given by (20) and (21) is constructed heuristically to synthesize several formerly reported scalings and patterns which are also displayed in our data: The square root scaling in (20) agrees with the results of Dentz et al (2004), which predict that $D_{\ell}(t) \propto \sqrt{t}$ in the pre-asymptotic regime (here, $t \ll t_{\mathrm{c}}$ ). In $(21), t_{\mathrm{c}}$ is proportional to the characteristic diffusive time $t_{\mathrm{d}}=\frac{1}{2} \mathcal{P}_{\mathrm{a}}$ for $\mathcal{P} \ll \mathcal{P}_{\mathrm{c}}$, and proportional to the advective time $t_{\mathrm{a}}$ for $\mathcal{P} \gg \mathcal{P}_{\mathrm{c}}$, in concurrence with Bijeljic and Blunt (2006). This means that sufficient sampling of the Eulerian velocity distribution by solute particles, which results in the stabilization of the dispersion coefficient (e.g., Dentz et al, 2000a,b), may be achieved with either diffusion or advection as the dominant mechanism, depending on the regime. Along these lines it is worth remarking that $\mathcal{P}_{\mathrm{c}} \gg 2$; this implies that diffusion remains an important velocity sampling mechanism even when transport at the microscopic (pore or grain) scale is advection-dominated. In other words, the "number of advective times" required by solute particles to sample the full distribution of Eulerian velocities by advection alone is much larger $\left(>100\right.$ times larger, considering that $\left.\mathcal{P}_{\mathrm{c}}>200\right)$ than the "number of diffusive times" required to do the same by diffusion alone. This is due to the high temporal correlation of fluid velocities along streamlines (specially in low-velocity regions, see de Anna et al (2013); Hakoun et al (2019)). The best-fit value of $\gamma$ in (21) for the mono-disperse medium is $\gamma=0.027$, implying that, for $\mathcal{P} \ll \mathcal{P}_{\mathrm{c}}$, the hydrodynamic term of the dispersion coefficient would reach $95 \%$ of its asymptotic value at $t \approx \frac{1}{2} t_{\mathrm{d}}$ (recall that characteristic times are defined based on grain, not pore, sizes). On the other hand, for $\mathcal{P} \gg \mathcal{P}_{\mathrm{c}}$, the same stage would be reached at $t \approx 55 t_{\mathrm{a}}$. Like $\mathcal{P}_{\mathrm{c}}$, the value of $\gamma$ appears to be sensitive to the heterogeneity of the medium (although in this case the three confidence intervals overlap considerably), as depicted in Figure 4(c). Hence, the critical time $t_{\mathrm{c}}$ required for dispersion stabilization increases with grain-size variability, especially for large $\mathcal{P}$ (when $t_{\mathrm{c}} \propto \mathcal{P}_{\mathrm{c}}$ ). This implies that it is even more difficult for the solute to sufficiently sample the velocity fluctuations by only advection in a poly-disperse medium than in the mono-disperse one, due to more pronounced preferential paths.

The time-dependence of the dispersion coefficient is not the only form of discrepancy between the observed transport and a purely Fickian behavior. Analysis of higher-order spatial moments, in particular $m^{(3)}$, reveals the emergence of a left skewness (i.e., a more pronounced rear than front tail) for high values of $\mathcal{P}$. The aforementioned work by Saffman (1960) includes an expression for $m^{(3)}(t)$ :

$$
\frac{m^{(3)}(t)}{\lambda^{3}}=-\frac{\mathcal{P}}{40} \frac{t}{t_{\mathrm{a}}}, \quad \text { for } 1 \ll \hat{\lambda} \mathcal{P} \ll 8 / \psi^{2},
$$

where $\lambda$ is the capillary length (equal to $0.78 d_{0}$ for the mono-disperse case, see above). Although the observed temporal evolution of $m^{(3)}(t)$ (not shown here for brevity) does roughly agree after some time with a constant growth as predicted by (22), this regime is preceded by an initial stage of slower growth (akin to the pre-asymptotic/asymptotic growth regimes of $m^{(2)}(t)$, discussed earlier) which is not captured by (22). Hence, this formula is arguably valid for large times only, similar to the asymptotic constant growth regime of $m^{(2)}(t)$ given by (16). By combining the two expressions we obtain the asymptotic relative plume skewness after Saffman,

$$
\hat{m}^{(3)}(t)=\frac{m^{(3)}}{\left[m^{(2)}\right]^{\frac{3}{2}}} \approx-\frac{\hat{\lambda}^{\frac{3}{2} \mathcal{P}}}{40\left[2 \tau^{-2} \hat{\lambda}^{-1} \mathcal{P}^{-1}+2 \beta(\mathcal{P})\right]^{\frac{3}{2}}}\left(\frac{t}{t_{\mathrm{a}}}\right)^{-\frac{1}{2}}, \quad \text { for } 1 \ll \hat{\lambda} \mathcal{P} \ll 8 / \psi^{2},
$$

with $\beta(\mathcal{P})$ being given by (19). The $\hat{m}^{(3)} \sim t^{-1 / 2}$ scaling, as well as the dependence of $\hat{m}^{(3)}$ on $\mathcal{P}$, are consistent with the well-established concepts that $(i)$ dispersion approaches a Fickian (and thus non-skewed) behavior as time progresses, and (ii) Fickian behavior is attained earlier for lower values of $\mathcal{P}$ (e.g, Dentz et al, 2004). Expression (23) is compared in Figure 4(d) to the relative plume skewness results at a late time $t \gg t_{\mathrm{a}}$ for all three cases of grain-size variability. Although the model slightly overestimates the magnitude of the observed plume skewness, it does correctly predict its sign and the approximate scaling with $\mathcal{P}$ within its applicability range. We also find that grain-size variability (the value of $\sigma$ ) does not have an obvious impact on the asymptotic relative plume skewness behavior.

From this discussion, we note that the attainment of a stable (asymptotic) dispersion coefficient does not necessarily imply true Fickian behavior. This is particularly evident for high values of $\mathcal{P}$, for which, at $t=141.6 t_{\mathrm{a}}$ 

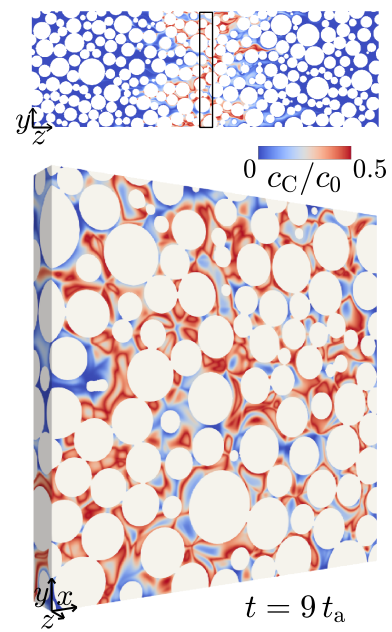

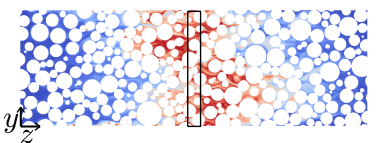

${ }^{0} c_{\mathrm{C}} / c_{0} 0.5$

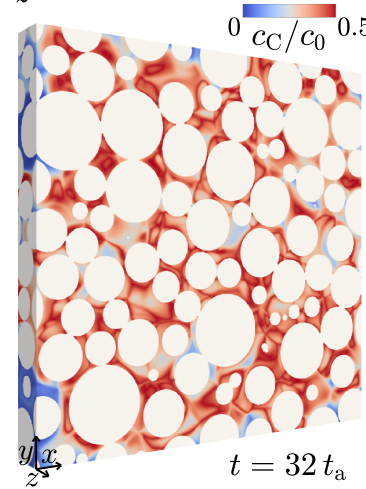

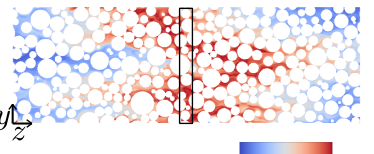

${ }^{0} c_{\mathrm{C}} / c_{0} 0.5$

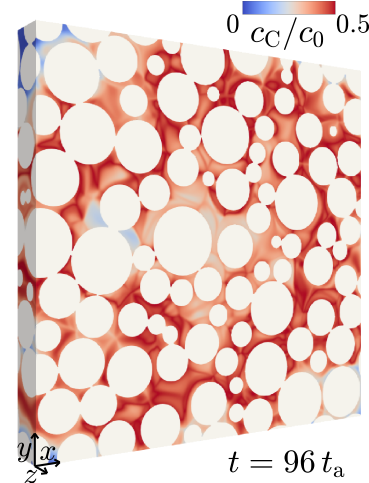

Fig. 5: Longitudinal cut view (top) and cross-section view at the moving interface (bottom) of pore-scale concentrations of chemical reaction product at three different times, with $\sigma=0.25$ and $\mathcal{P}=1155$. Mixing causes the concentrations of reaction product at and near the interface to increase over time.

with $\sigma=0, D_{\ell}$ is visibly constant in time (see Figure $4(\mathrm{~b})$ ) but the plume is notably skewed, as shown by $\hat{m}^{(3)}$ (see Figure 4(d)).

\subsection{Reactive transport}

Let us now consider a chemical reaction involving two reactants $\mathrm{A}$ and $\mathrm{B}$, which form a reaction product $\mathrm{C}$,

$$
\mathrm{A}+\mathrm{B} \rightarrow \mathrm{C} .
$$

By simple use of the stoichiometry, one may define two chemically conservative components, $\mathrm{A}^{\prime}$ and $\mathrm{B}^{\prime}$, whose concentration values are insensitive to the reaction (24):

$$
c_{\mathrm{A}^{\prime}}=c_{\mathrm{A}}+c_{\mathrm{C}}, \quad c_{\mathrm{B}^{\prime}}=c_{\mathrm{B}}+c_{\mathrm{C}} .
$$

Let us assume that $(i)$ all chemical species are transported by advection-diffusion with the same diffusion coefficient $D$, and ( $i i)$ the chemical reaction is irreversible and instantaneous. Then, $c_{\mathrm{A}^{\prime}}$ and $c_{\mathrm{B}^{\prime}}$ also obey the advection-diffusion equation with diffusion coefficient $D$ and, since A and B cannot (locally) coexist, the concentration of product C can be determined at any time and position by:

$$
c_{\mathrm{C}}(\mathbf{x}, t)=\min \left(c_{\mathrm{A}^{\prime}}(\mathbf{x}, t), c_{\mathrm{B}^{\prime}}(\mathbf{x}, t)\right) .
$$

The values of $c(\mathbf{x}, t)$ resulting from the simulations described in $\S 2$ can be re-interpreted to represent the transport and mixing-driven chemical reaction between two solutions carrying reactants $\mathrm{A}$ and $\mathrm{B}$ respectively, both with initial concentration $c_{0}$, and initially separated by a sharp interface at $z=0$. This only requires defining:

$$
c_{\mathrm{A}^{\prime}}(\mathbf{x}, t)=c(\mathbf{x}, t), \quad c_{\mathrm{B}^{\prime}}(\mathbf{x}, t)=c_{0}-c(\mathbf{x}, t) .
$$

Figure 5 corresponds to the same conditions $\left(\sigma=0.25, \mathcal{P} \approx 10^{3}\right)$, regions and times of Figure 3 , but in this case we show the pore-scale concentrations of reaction product C. Local segregation between the two reactants gradually fades over time, generating an increasing amount of reaction product within pores. 
Below, the observed coarse-scale behavior of reaction product $\mathrm{C}$ is analyzed. In $\$ 3.2 .1$, the under-production of $\mathrm{C}$ due to local mixing limitation is studied for different Péclet numbers and degrees of material heterogeneity. In $\S 3.2 .2$, the direct influence of local concentration fluctuations on hypothetical colorimetric observations is explored, based on data from the physical experiment of Gramling et al (2002).

\subsubsection{Observations of mixing-limited reaction}

As noted above, $\mathrm{A}^{\prime}$ and $\mathrm{B}^{\prime}$ are chemically conservative. One may then approximate their coarse-scale onedimensional transport with the hydrodynamic advection-dispersion equation:

$$
\frac{\partial \bar{c}_{I}}{\partial t}=-u \frac{\partial \bar{c}_{I}}{\partial z}+D_{\ell}(t) \frac{\partial^{2} \bar{c}_{I}}{\partial z^{2}}, \quad I=\mathrm{A}^{\prime}, \mathrm{B}^{\prime},
$$

where $D_{\ell}(t)$ may either be assigned a time-dependent or a constant asymptotic value (see $\S 3.1$ ). The solution of (28), given the initial conditions and for remote boundaries, is

$$
\bar{c}_{\mathrm{A}^{\prime}}(z, t)=c_{0}-\bar{c}_{\mathrm{B}^{\prime}}(z, t)=\frac{c_{0}}{2} \operatorname{erfc}\left(\frac{z-u t}{2 \sqrt{\int_{0}^{t} D_{\ell}(\tau) \mathrm{d} \tau}}\right) \equiv \frac{c_{0}}{2} \operatorname{erfc}\left(\frac{z-u t}{\sqrt{2 m^{(2)}(t)}}\right) .
$$

Given solution (29), assuming complete mixing over an averaging volume (which we denote as well-mixed conditions) one would then predict, from (26),

$$
\bar{c}_{\mathrm{C}}^{\mathrm{wm}}(z, t)=\min \left(\bar{c}_{\mathrm{A}^{\prime}}(z, t), \bar{c}_{\mathrm{B}^{\prime}}(z, t)\right)=\frac{c_{0}}{2} \operatorname{erfc}\left(\frac{|z-u t|}{\sqrt{2 m^{(2)}(t)}}\right),
$$

and the amount of mass produced by the reaction $\left(\mathrm{MT}^{-1}\right)$ would be

$$
M_{\mathrm{C}}^{\mathrm{wm}}(t)=n S \int \bar{c}_{\mathrm{C}}^{\mathrm{wm}}(z, t) \mathrm{d} z=n S c_{0} \sqrt{2 m^{(2)}(t) / \pi},
$$

where $S$ is the cross-section area (here $S=81 d_{0}^{2}$ ).

Concentration fluctuations over spatial scales below the size of an averaging volume (pore-scale fluctuations) induce a decrease in the amount of actual reaction with respect to the prediction by (30) and (31). Hence, observations of the actual amount of reaction indirectly quantify the departure of the actual system from well-mixed conditions (i.e., the incomplete mixing).

Figure 6(a) shows the comparison between the actual product concentrations $c_{\mathrm{C}}$ and the ideal well-mixed prediction $c_{\mathrm{C}}^{\mathrm{wm}}$ (equation (30)), in the mono-disperse scenario, for $\mathcal{P}=10^{3.5}$ and $\mathcal{P}=10^{1}$, along $z$ at three different times. As one might expect, the degree of departure of $c_{\mathrm{C}}$ from $c_{\mathrm{C}}^{\mathrm{wm}}$ is more marked for the higher value of $\mathcal{P}$. Additionally, careful observation of the concentration peaks and tails for $\mathcal{P}=10^{3.5}$ reveals that the shape of $c_{\mathrm{C}}$ is asymmetric. This feature is not observable for $\mathcal{P}=10$. Both these results are consistent with the dependence of longitudinal skewness on $\mathcal{P}$ analyzed for nonreactive transport in $\S 3.1$ (see Figure 4(d)).

Figure 6(b) depicts the temporal evolution of the departure of the generated reaction product mass, $M_{\mathrm{C}}$, from the ideal well-mixed solution $M_{\mathrm{C}}^{\mathrm{wm}}$ (equation (31)), expressed as $1-M_{\mathrm{C}} / M_{\mathrm{C}}^{\mathrm{wm}}$. This metric is used to globally quantify the degree of incomplete mixing and reaction. Results are displayed for the mono-disperse case $(\sigma=0)$ and for various values of $\mathcal{P}$ (line color intensity). The behavior of this metric with time is relatively close to a power law (i.e., close to a straight line on a log-log scale). Increasing the Péclet number $\mathcal{P}$ produces an upward shift of the curve in the log-log space, as well as a (slight) reduction in slope.

When comparing the same set of results among the different materials (characterized by different grain-size variability $\sigma$ but with identical mean hydraulic radius and typical - i.e. modal - pore size), we find (see Figure 6(d)) that the results for $1-M_{\mathrm{C}} / M_{\mathrm{C}}^{\mathrm{wm}}$ as a function of $t / t_{\mathrm{a}, 0}$, where $t_{\mathrm{a}, 0}=d_{0} / u$, appear to be quite similar among different materials for matching Péclet numbers based on $d_{0}$, i.e., $\mathcal{P}_{0}=u d_{0} / \mathcal{P}$. That is, if we set the characteristic length to 

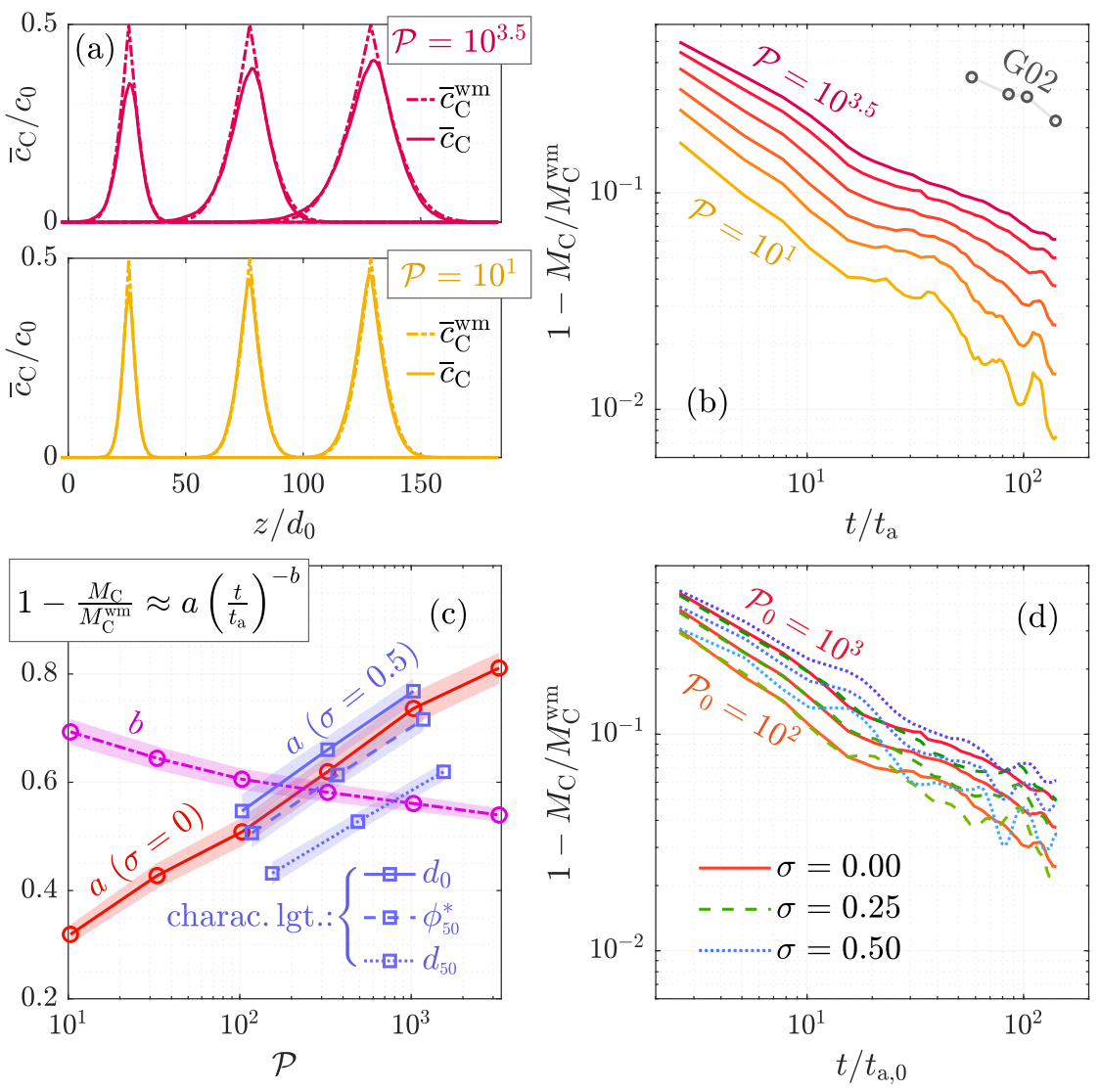

Fig. 6: (a) Measured longitudinal concentration profiles of reaction product (solid lines) at times $25.7 t_{\mathrm{a}}, 77.2 t_{\mathrm{a}}$ and $128.7 t_{\mathrm{a}}$, compared to the ideal well-mixed behavior (dash-dotted lines, equation (30)), for the mono-disperse case ( $\sigma=0)$ with $\mathcal{P}=10^{3.5}$ (top) and $\mathcal{P}=10$ (bottom); (b) Temporal evolution of the relative degree of incomplete reaction $1-M_{\mathrm{C}} / M_{\mathrm{C}}^{\mathrm{wm}}$ for the mono-disperse case $(\sigma=0)$ with $\log \mathcal{P}$ ranging from 1 to 3.5 at 0.5 intervals, and comparison to the G02 experiment (circles); (c) Best power-law parameter fits of the curves shown in (b) and (c), given by coefficients $b$ (does not change with $\sigma$ ) and $a$ (red for $\sigma=0$; blue for $\sigma=0.5$, with three alternative definitions of characteristic length in $\mathcal{P}$ and $t_{\mathrm{a}}$, where $\left.\phi_{50}^{*}=d_{0} \phi_{50} / \phi_{50,0}\right)$; and (d) Same as (b), but including the three degrees of grain size variability $(\sigma)$, and with $\log \mathcal{P}_{0}$ ranging from 2 to 3 , where $\mathcal{P}_{0}=u d_{0} / D$.

be $d_{0}$ (which is proportional to the hydraulic radius), besides the understandable noise, one cannot clearly conclude from the data that the incomplete mixing and reaction increases or decreases with increasing $\sigma$. This suggests that the hydraulic radius, or equivalently $d_{0}$, may be a characteristic length that unifies the mixing behaviors of the three media (similar to the case of permeability, see \$2.2.2).

In order to confirm this hypothesis, we determine parameter values $a$ and $b$, and corresponding $95 \%$ confidence bounds, for the curves depicted in Figures 6(b,d) corresponding to $\sigma=0$ and $\sigma=0.5$, given the assumption that $1-M_{\mathrm{C}} / M_{\mathrm{C}}^{\mathrm{wm}} \approx a\left(t / t_{\mathrm{a}}\right)^{-b}$, while testing different possible choices of characteristic length for the poly-disperse medium. The results are shown in Figure 6(c). For $b$ (the power-law slope), all curves display equivalent (i.e., very strongly overlapping) behavior with $\mathcal{P}$, and therefore for the sake of clarity we only show the result for $\sigma=0$. Coefficient $b$ diminishes as $\mathcal{P}$ increases, and it appears to approach $b \approx 0.5$ for very high $\mathcal{P}$. With respect to $a$, results show that the curves corresponding to $\sigma=0$ and to $\sigma=0.5$ are indeed very close when $d_{0}$ is the characteristic length, with considerable overlap between their respective $95 \%$ confidence intervals. This unification 
between the mixing behaviors of the different media is possibly even more evident when using a characteristic length that is proportional to the volume-weighted median pore diameter, $\phi_{50}^{*}=d_{0} \phi_{50} / \phi_{50,0}$ (where $\phi_{50,0}$ is the value of $\phi_{50}$ for $\left.\sigma=0\right)$. On the other hand, for the default choice of characteristic length $d_{50}$ (which does control singlespecies asymptotic dispersion, see §3.1), there is a clear disagreement between the two curves. This reinforces the hypothesis that the typical pore size and/or the mean hydraulic radius (which could be inferred from grain distribution information) are proper characteristic lengths associated with (incomplete) mixing, which is locally controlled by the rate of concentration homogenization within pores. It is also worth recalling that, for the kinds of granular media considered, we found that the mean hydraulic radius could potentially be inferred from the observed permeability and porosity (see $\$ 2.2 .2)$.

\subsubsection{Impact of local concentration variability on measurements}

Figure 6(b), as discussed in $\$ 3.2 .1$, shows the temporal evolution of incomplete reaction at different Péclet numbers, and also includes the results of Gramling et al (2002) (hereafter referred to as G02), with values calculated by Alhashmi et al (2015) from the data. In G02, an experimental porous medium column (with dimensions $\left.13.8 d_{0} \times 42.3 d_{0} \times 277 d_{0}\right)$ was initially filled with solution B $\left(\mathrm{EDTA}^{4-}\right)$ and then flushed by solution $\mathrm{A}\left(\mathrm{CuSO}_{4}\right)$. When in contact, they form product $\mathrm{C}\left(\mathrm{CuEDTA}^{2-}\right)$ through a virtually instantaneous and irreversible chemical reaction. This is essentially equivalent to the configuration of our computational model. The granular material was not made of spheres, but instead of cryolite sand with fairly uniform grain sizes averaging $d_{0}=0.13 \mathrm{~cm}$. According to the authors of the experiment, the results of G02 shown in Figure 6(b) correspond to a grain Péclet number of $\mathcal{P}=10^{3.35}$, which is very similar to the highest value of $\mathcal{P}=10^{3.5}$ tested here. Yet, the observed degree of incomplete reaction is ostensibly larger than in our results. Multiple factors (or a combination of them) could explain this discrepancy, including the subtle differences in initial and boundary conditions at the interface (Gurung and Ginn, 2020), the possible influence of the lateral walls of the column on the flow and transport in G02, or the irregular geometry of the grains (as opposed to the spherical grains employed here). Below we explore the possible influence on the results of yet another element.

Available observations in G02 are light absorbance values inferred from pixel color intensity in photographic images. These are therefore pixel-scale (i.e, coarse or continuum-scale) observations. Averaging in the physical experiment may occur in at least four ways, namely $(i)$ intended physical averaging by light traveling in the direction orthogonal to the camera lens, (ii) unintended non-orthogonal physical averaging, due to the non-orthogonality of light beams, refraction within cryolite grains, light scattering and light dispersion, (iii) limited resolution of the images (pixel size), and (iv) post-process averaging, by integrating multiple pixels into a single value. In light of this we will discuss, qualitatively and quantitatively, the combined effect of $(i)$ local concentration fluctuations, $(i i)$ coarse-scale observations involving volume averaging, and (iii) a non-linear relation between solute concentration and measured absorbance. The combination of these factors could result in a disagreement between the coarse-scale concentrations of the solute (in this case, the reaction product) inferred from observed light intensities, and the actual concentrations. Note that this is a different effect, akin to experimental error, than the actual slow-down of reaction product generation due to incomplete mixing discussed in \$3.2.1.

Unfortunately, there is some ambiguity in the manuscript by Gramling et al (2002) regarding the concentrationabsorbance relationship: Although Beer-Lambert's law is mentioned, it is unclear whether the apparent molar absorptivity was constant, and the original "empirically determined calibration curve between known concentrations of CuEDTA ${ }^{2-}$ and their observed light absorbance" is not available. Yet, the comparison between concentration data extracted from the cited paper and the original absorbance data shared by the authors provides some evidence that the calibration curve was probably nonlinear, as shown in Figure 7(a), with the apparent absorbance exhibiting a concave non-linear behavior with respect to concentration, instead of the ideal Beer-Lambert proportionality. The data are well fit by

$$
\frac{A}{A_{\text {ref }}}=g(c)=\kappa \varepsilon\left[1-\exp \left(-\frac{c}{\varepsilon}\right)\right],
$$

where $A_{\text {ref }}$ is the maximum absorbance datum, emulating saturation for $c \gtrsim \varepsilon$, with $\varepsilon=(0.3146 \pm 0.0270) c_{0}$ and $\kappa=(4.624 \pm 0.141) c_{0}^{-1}$. Errors in the digitizing process probably contribute to the visible noise around the trend. 
The apparent concave deviation from Beer-Lambert's law (due, in fact, to a non-logarithmic relationship between light intensity and absorbance) is not unusual, and it has also been observed in other porous media experiments (de Anna et al, 2014, supporting information). It may be explained by several factors, including heterogeneity of the refractive index and/or concentrations above the applicability threshold (Mayerhöfer et al, 2016; Tolbin et al, 2018).

The calibrated curve $g(c)$ describes the apparent absorbance behavior when concentrations are constant across the column, but it may not hold in the presence of local pore-scale fluctuations. To address the effect that these fluctuations may have on the measurements, we make the simplifying assumption that all points in the porous space contribute equally and additively to the overall apparent absorbance (i.e., we neglect possible non-local sources of apparent absorbance non-linearity), and hence the same curve $g(c)$ applies locally to each individual point. Then, the concentration that one would infer in a pixel-size averaging volume is

$$
\bar{c}^{\mathrm{obs}}=g^{-1}(\overline{g(c)})
$$

which may differ from the volume-averaged concentration $\bar{c}$ if $g(c)$ is nonlinear, i.e., if $\overline{g(c)} \neq g(\bar{c})$. Although the assumptions adopted in (33) undoubtedly constitute an oversimplification, this simple exercise provides a qualitative assessment of possible disagreements between reality and observations due to a calibration curve like (32). Let us approximate the local variability of concentrations within an averaging volume $\Omega(z)$ with a Gaussian distribution,

$$
f(c)=(2 \pi \Sigma)^{-\frac{1}{2}} \exp \left(\frac{[c-\bar{c}]^{2}}{2 \Sigma}\right)
$$

where $\Sigma=\overline{(c-\bar{c})^{2}}$ is the local concentration variance. Then,

$$
\overline{g(c)}=\int f(c) g(c) \mathrm{d} c=\kappa \varepsilon\left[1-\exp \left(-\frac{\bar{c}-[2 \varepsilon]^{-1} \Sigma}{\varepsilon}\right)\right] .
$$

This, combined with (33), implies that $\bar{c}^{\mathrm{obs}}=\bar{c}-[2 \varepsilon]^{-1} \Sigma$; that is, there is an underestimation of $\bar{c}$ which is proportional to variance $\Sigma$.

To be more concrete, we may now look at the difference between the actual concentration of product $\bar{c}_{\mathrm{C}}$ and the "observed" $\bar{c}_{\mathrm{C}}^{\text {obs }}$ calculated via (33) in our computational experiment. The comparison is shown for $\mathcal{P}=10^{3.5}$ in Figure $7(\mathrm{~b})$, together with the well-mixed $\bar{c}_{\mathrm{C}}^{\mathrm{wm}}$ for reference, showing that the decrease in concentration product with respect to the ideal $\bar{c}_{\mathrm{C}}^{\mathrm{wm}}$ is overestimated by $c_{\mathrm{C}}^{\mathrm{obs}}$ because of the non-linear concentration-absorbance relationship. While not dramatic, this effect is by no means negligible. It is also worth noting that the region where product concentrations differ from the well-mixed scenario appears to be significantly wider for $\bar{c}_{\mathrm{C}}^{\mathrm{obs}}$ than it actually is for $\bar{c}_{\mathrm{C}}$. Thus, under the present assumptions, observations of the reaction product plume based on light intensity exaggerate the actual extent of longitudinal narrowing induced by mixing limitation. We may also examine the difference in the degree of incomplete reaction quantified by $1-M_{\mathrm{C}} / M_{\mathrm{C}}^{\mathrm{wm}}$ (Figure 7 (c)), calculated from the true and the observed concentrations (solid and dotted line, respectively) with $\mathcal{P}=10^{3.5}$. For reference, the line corresponding to $\mathcal{P}=10^{2.5}$ is also shown, as well as the data from G02 (reportedly with $\mathcal{P}=10^{3.35}$ ). One of the main takeaways is that the magnitude of the observation error is comparable to - if not larger than - the effect of increasing the Péclet number by one order of magnitude. Furthermore, while still considerably lower than the G02 results, the observed degree of incomplete reaction (dotted line) is visibly closer to G02 than the true values (solid line) are. This suggests that, while mostly overlooked to date, error caused by volume averaging in the light signal may artificially amplify the reported effects of incomplete mixing in the G02 experiments. As mentioned at the beginning of this section, other factors may also contribute to the discrepancy between our observations and those of G02, including lateral boundary effects, grain geometries and nuances in the initial condition. 

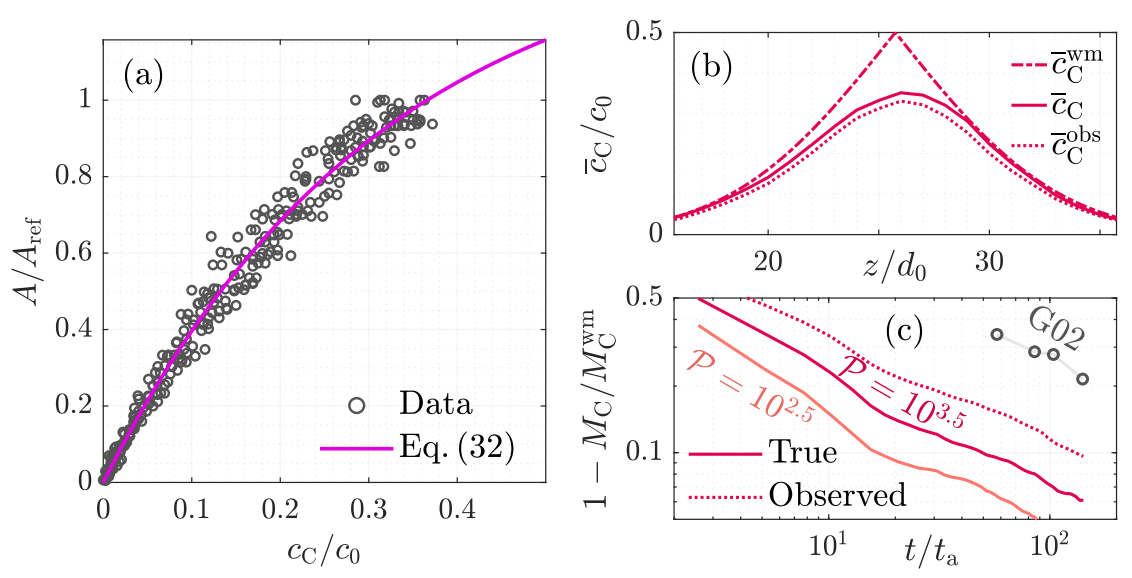

Fig. 7: (a) Concentration-absorbance relationship in G02, reconstructed by comparing original absorbance data with the reported concentrations in the paper; (b) Longitudinal coarse-scale concentration profiles of reaction

product in our simulations for the mono-disperse case $(\sigma=0)$ with $\mathcal{P}=10^{3.5}$ at $t=25.7 t_{\mathrm{a}}$ comparing the well-mixed case $\bar{c}_{\mathrm{C}}^{\mathrm{wm}}$, the actual concentrations $\bar{c}_{\mathrm{C}}$ and the apparent concentrations $\bar{c}_{\mathrm{C}}^{\mathrm{obs}}$ interpreted from light absorbance (equation (33)); and (c) Temporal evolution of the relative degree of incomplete reaction $1-M_{\mathrm{C}} / M_{\mathrm{C}}^{\mathrm{wm}}$ for the mono-disperse case $(\sigma=0)$ with $\mathcal{P}=10^{3.5}$ (solid red) and with $\mathcal{P}=10^{2.5}$ (solid orange), compared to the observed values interpreted from light absorbance with $\mathcal{P}=10^{3.5}$ (dotted red), as well as the G02 data (circles).

\section{Summary and conclusions}

We have presented a set of extreme-resolution pore-scale flow and transport simulations emulating a long (nearly 200 grain diameters) column experiment to study macroscopic dispersion, mixing and reaction in granular porous media under laminar saturated flow conditions. The media, built through gravitational settling of spherical solid grains, are generated with various degrees of grain-size variability but designed to have the same expected mean hydraulic radius (i.e., the same specific surface). We see that, as a result, they exhibit $(i)$ equal mode in the pore size distribution, and ( $\mathrm{ii}$ ) nearly identical values of permeability divided by porosity. Furthermore, the permeability value is very closely predicted from the hydraulic radius (which is a known function of the grain-size distribution) by the Kozeny-Carman equation.

The very demanding flow (Navier-Stokes) and transport (Advection-Diffusion) simulations were conducted at the Barcelona Supercomputing Center (see Acknowledgments) by taking advantage of the massively parallel scalability of the OpenFOAM suite. The transport simulations explore a range of Péclet numbers corresponding to advection-dominated conditions at the pore scale. Hydrodynamic dispersion behavior and its relation to the Péclet number is in very close agreement with classical theories and existing experimental observations, as well as with the common notion that, in the long-time and large-Péclet limits, a granular medium's effective hydrodynamic dispersivity is approximately equal to the volume-weighted median grain diameter. Increased grain-size variability results in higher values of the critical Péclet number (which is the Péclet number above which the asymptotic longitudinal dispersion coefficient is proportional to the mean fluid velocity), and in a longer time required for reaching the asymptotic dispersion regime. Analysis of the plume skewness shows that a stable dispersion coefficient does not necessarily indicate Fickian behavior.

The same set of simulations may be re-interpreted as a reactive transport experiment where two solutions, initially separated by a sharp interface that is normal to the mean fluid velocity, mix and react as they advance through the column. Due to local incomplete mixing at the pore scale, coarse-scale concentrations of reaction product are lower than one would predict by assuming locally well-mixed conditions. This effect is stronger for higher Péclet numbers, which also produce more asymmetric (skewed) concentration profiles. Within our range of 
conditions, the degree of grain-size variability does not seem to significantly impact the ratio between global reaction product mass and the ideal well-mixed prediction. Instead, a Péclet number based on the typical pore size and/or the mean hydraulic radius appears to largely control the temporal evolution of this metric. Finally, we explore the possible discrepancy between true and observed reaction product concentrations when these are inferred from light intensity signal and a non-linear concentration-absorbance calibration curve, as evidence suggests to be the case of the Gramling et al (2002) experiments. We show that the concavity of the curve may lead to an error in the coarsescale reaction product concentration measurement that is (under some simplifying assumptions) proportional to the concentration variance arising from pore-scale fluctuations. Applied directly to our computational experiments, the non-linear calibration curve would result in a considerable underestimation of the true coarse-scale reaction product concentration, thus mimicking the actual incomplete mixing effect of increasing the Péclet number by one order of magnitude.

Through the part of this study that refers to the work by Gramling et al (2002), we do not wish in the least to diminish the invaluable experiments conducted by the authors, but rather urge the community at large to be cautious in taking these data too far as well as urging experimentalists to conduct further essential high quality laboratory and numerical experiments with which to unravel the fascinating story of incomplete mixing and reactions in porous media.

The set of high-resolution simulation results does not only provide new insights on transport behavior as reported within this paper; it also gives unprecedentedly privileged simultaneous access to both continuum-scale observations and microscopic fluid flow and solute transport data, for the scientific community to further study fundamental processes in the future. We are committed to sharing these data openly (see Data availability). Future research avenues that we envisage include $(i)$ An in-depth analysis of the pore-scale velocity statistics and how these are linked to solute transport, and (ii) fully describing and analyzing the transport and evolution of the solute concentration's probability density function over space and time, as well as evaluating the ability of existing (or new) transport-mixing models (e.g., Suciu et al, 2015; Sole-Mari et al, 2020) to predict its behavior.

Acknowledgements The authors thankfully acknowledge the computer resources at MareNostrum and the technical support provided by the Barcelona Supercomputing Center (research activity AECT-2019-3-0014). D. B. acknowledges financial support by the U.S. Army Research Office under Contract/Grant W911NF-18-1-0338. D. F. acknowledges financial support by the European Commission through project MARSOLUT (H2020-MSCA-ITN-2018), and by the Spanish Ministry of Science, Innovation and Universities through project MONOPOLIOS (RTI2018-101990-B-I00). The authors thankfully acknowledge Charles F. Harvey for sharing the G02 light absorbance data.

\section{Declarations}

Funding This work was partially funded by the U.S. Army Research Office (W911NF-18-1-0338), the European Commission (H2020MSCA-ITN-2018), and the Spanish Ministry of Science, Innovation and Universities (RTI2018-101990-B-I00).

Data availability The processed coarse-scale data analyzed within this paper, as well as instructions for downloading the original micro-scale results dataset, are available on Zenodo (https://doi .org/10.5281/zenodo.4925044).

Code availability The presented simulations were performed using the open-source software OpenFOAM.

Conflicts of interest The authors declare that they have no conflict of interest.

\section{References}

Alhashmi Z, Blunt M, Bijeljic B (2015) Predictions of dynamic changes in reaction rates as a consequence of incomplete mixing using pore scale reactive transport modeling on images of porous media. Journal of Contaminant Hydrology 179:171 - 181, doi:10.1016/j.jconhyd.2015.06.004

de Anna P, Le Borgne T, Dentz M, Tartakovsky AM, Bolster D, Davy P (2013) Flow intermittency, dispersion, and correlated continuous time random walks in porous media. Physical review letters 110(18):184,502, doi:10.1103/PhysRevLett.110.184502 
de Anna P, Jimenez-Martinez J, Tabuteau H, Turuban R, Le Borgne T, Derrien M, Méheust Y (2014) Mixing and reaction kinetics in porous media: An experimental pore scale quantification. Environmental Science \& Technology 48(1):508-516, doi:10.1021/es403105b

Aris R, Taylor GI (1956) On the dispersion of a solute in a fluid flowing through a tube. Proceedings of the Royal Society of London Series A Mathematical and Physical Sciences 235(1200):67-77, doi:10.1098/rspa.1956.0065

Bear J (1972) Dynamics of Fluids in Porous Media. Elsevier, New York

Benson DA, Meerschaert MM (2008) Simulation of chemical reaction via particle tracking: Diffusion-limited versus thermodynamic rate-limited regimes. Water Resources Research 44(12), doi:10.1029/2008WR007111

Bijeljic B, Blunt MJ (2006) Pore-scale modeling and continuous time random walk analysis of dispersion in porous media. Water Resources Research 42(1), doi:10.1029/2005WR004578

Bijeljic B, Muggeridge AH, Blunt MJ (2004) Pore-scale modeling of longitudinal dispersion. Water Resources Research 40(11), doi:10.1029/2004WR003567

Bolster D, Barahona M, Dentz M, Fernàndez-Garcia D, Sanchez-Vila X, Trinchero P, Valhondo C, Tartakovsky DM (2009) Probabilistic risk analysis of groundwater remediation strategies. Water Resources Research 45(6), doi:10.1029/2008WR007551

Carman PC (1937) Fluid flow through granular beds. Transactions of the Institution of Chemical Engineers 15:155166, doi:10.1016/S0263-8762(97)80003-2

Chiogna G, Bellin A (2013) Analytical solution for reactive solute transport considering incomplete mixing within a reference elementary volume. Water Resources Research 49(5):2589-2600, doi:10.1002/wrcr.20200

Dentz M, Kinzelbach H, Attinger S, Kinzelbach W (2000a) Temporal behavior of a solute cloud in a heterogeneous porous medium: 1. point-like injection. Water Resources Research 36(12):3591-3604, doi:10.1029/2000WR900162

Dentz M, Kinzelbach H, Attinger S, Kinzelbach W (2000b) Temporal behavior of a solute cloud in a heterogeneous porous medium: 2. spatially extended injection. Water Resources Research 36(12):3605-3614, doi:10.1029/2000WR900211

Dentz M, Cortis A, Scher H, Berkowitz B (2004) Time behavior of solute transport in heterogeneous media: transition from anomalous to normal transport. Advances in Water Resources 27(2):155 - 173, doi:10.1016/j.advwatres.2003.11.002

Dentz M, Le Borgne T, Englert A, Bijeljic B (2011) Mixing, spreading and reaction in heterogeneous media: A brief review. doi:10.1016/j.jconhyd.2010.05.002

Ding D, Benson DA, Paster A, Bolster D (2013) Modeling bimolecular reactions and transport in porous media via particle tracking. Advances in Water Resources 53:56 - 65, doi:10.1016/j.advwatres.2012.11.001

Dybas MJ, Hyndman DW, Heine R, Tiedje J, Linning K, Wiggert D, Voice T, Zhao X, Dybas L, Criddle CS (2002) Development, operation, and long-term performance of a full-scale biocurtain utilizing bioaugmentation. Environmental science \& technology 36(16):3635-3644, doi:10.1021/es0114557

Edery Y, Scher H, Berkowitz B (2009) Modeling bimolecular reactions and transport in porous media. Geophysical Research Letters 36(2), doi:10.1029/2008GL036381

Finney JL, Bernal JD (1970) Random packings and the structure of simple liquids. i. the geometry of random close packing. Proceedings of the Royal Society of London A Mathematical and Physical Sciences 319(1539):479-493, doi:10.1098/rspa.1970.0189

Gardner WR (1956) Representation of soil aggregate-size distribution by a logarithmic-normal distribution. Soil Science Society of America Journal 20(2):151-153, doi:10.2136/sssaj1956.03615995002000020003x

Ginn TR (2018) Modeling bimolecular reactive transport with mixing-limitation: Theory and application to column experiments. Water Resources Research 54(1):256-270, doi:10.1002/2017WR022120

Gramling CM, Harvey CF, Meigs LC (2002) Reactive Transport in Porous Media: A Comparison of Model Prediction with Laboratory Visualization. Environmental Science \& Technology 36(11):2508-2514, doi:10.1021/es0157144

Gurung D, Ginn TR (2020) Mixing ratios with age: Application to preasymptotic one-dimensional equilibrium bimolecular reactive transport in porous media. Water Resources Research 56(7):e2020WR027,629, doi:10.1029/2020WR027629 
Hakoun V, Comolli A, Dentz M (2019) Upscaling and prediction of lagrangian velocity dynamics in heterogeneous porous media. Water Resources Research 55(5):3976-3996, doi:10.1029/2018WR023810

Heyman J, Lester DR, Turuban R, Méheust Y, Le Borgne T (2020) Stretching and folding sustain microscale chemical gradients in porous media. Proceedings of the National Academy of Sciences 117(24):13,359-13,365, doi:10.1073/pnas.2002858117

Hiby JW (1959) Longitudinal and transverse mixing during single-phase flow through granular beds. In: Conf. Dispersion Porous Media, Imperial College, London

Hochstetler DL, Kitanidis PK (2013) The behavior of effective rate constants for bimolecular reactions in an asymptotic transport regime. Journal of Contaminant Hydrology 144(1):88 - 98, doi:10.1016/j.jconhyd.2012.10.002

Icardi M, Boccardo G, Marchisio DL, Tosco T, Sethi R (2014) Pore-scale simulation of fluid flow and solute dispersion in three-dimensional porous media. Phys Rev E 90:013,032, doi:10.1103/PhysRevE.90.013032

Jaeger HM, Nagel SR (1992) Physics of the granular state. Science 255(5051):1523-1531, doi:10.1126/science.255.5051.1523

Koponen A, Kataja M, Timonen J (1996) Tortuous flow in porous media. Phys Rev E 54:406-410, doi:10.1103/PhysRevE.54.406

Kozeny M (1927) Uber kapillare leitung des wassers im boden. Sitzungsber Akad Wiss Wien 136(2a):271-306

Le Borgne T, Dentz M, Davy P, Bolster D, Carrera J, de Dreuzy JR, Bour O (2011) Persistence of incomplete mixing: A key to anomalous transport. Phys Rev E 84:015,301, doi:10.1103/PhysRevE.84.015301

Mayerhöfer TG, Mutschke H, Popp J (2016) Employing theories far beyond their limits- the case of the (boguer-) beer-lambert law. ChemPhysChem 17(13):1948-1955, doi:10.1002/cphc.201600114

van Milligen BP, Bons PD (2012) Analytical model for tracer dispersion in porous media. Phys Rev E 85:011,306, doi:10.1103/PhysRevE.85.011306

Oates P (2007) Upscaling reactive transport in porous media: laboratory visualizations and stochastic models. $\mathrm{PhD}$ thesis, Massachusetts Institute of Technology

Paster A, Bolster D, Benson DA (2013) Particle tracking and the diffusion-reaction equation. Water Resources Research 49(1):1-6, doi:10.1029/2012WR012444

Paster A, Bolster D, Benson DA (2014) Connecting the dots: Semi-analytical and random walk numerical solutions of the diffusion-reaction equation with stochastic initial conditions. Journal of Computational Physics 263:91112, doi:10.1016/j.jcp.2014.01.020

Pfannkuch HO (1963) Contribution à l'étude des déplacements de fluides miscibles dans un milieu poreux. Rev Inst Fr Petrole 18:215 - 270

Porta G, Ceriotti G, Thovert JF (2016) Comparative assessment of continuum-scale models of bimolecular reactive transport in porous media under pre-asymptotic conditions. Journal of Contaminant Hydrology 185-186:1 - 13, doi:10.1016/j.jconhyd.2015.12.003

Raje DS, Kapoor V (2000) Experimental study of bimolecular reaction kinetics in porous media. Environmental Science \& Technology 34(7):1234-1239, doi:10.1021/es9908669

Saffman PG (1960) Dispersion due to molecular diffusion and macroscopic mixing in flow through a network of capillaries. Journal of Fluid Mechanics 7(2):194-208, doi:10.1017/S0022112060001432

Sahimi M (2011) Flow and Transport in Porous Media and Fractured Rock: From Classical Methods to Modern Approaches, Second Edition. Wiley-VCH, doi:10.1002/9783527636693

Sanchez-Vila X, Fernàndez-Garcia D, Guadagnini A (2010) Interpretation of column experiments of transport of solutes undergoing an irreversible bimolecular reaction using a continuum approximation. Water Resources Research 46(12), doi:10.1029/2010WR009539

Schmidt MJ, Pankavich S, Benson DA (2017) A Kernel-based Lagrangian method for imperfectly-mixed chemical reactions. Journal of Computational Physics 336:288-307, doi:10.1016/j.jcp.2017.02.012

Schmidt MJ, Pankavich SD, Benson DA (2018) On the accuracy of simulating mixing by random-walk particle-based mass-transfer algorithms. Advances in Water Resources 117:115 - 119, doi:10.1016/j.advwatres.2018.05.003

Schmidt MJ, Pankavich SD, Navarre-Sitchler A, Benson DA (2019) A lagrangian method for reactive transport with solid/aqueous chemical phase interaction. Journal of Computational Physics: X 2:100,021, doi:10.1016/j.jcpx.2019.100021 
Scott GD, Kilgour DM (1969) The density of random close packing of spheres. Journal of Physics D: Applied Physics 2(6):863-866, doi:10.1088/0022-3727/2/6/311

Silin D, Patzek T (2006) Pore space morphology analysis using maximal inscribed spheres. Physica A: Statistical Mechanics and its Applications 371(2):336-360, doi:10.1016/j.physa.2006.04.048

Sole-Mari G, Fernàndez-Garcia D, Sanchez-Vila X, Bolster D (2020) Lagrangian modeling of mixing-limited reactive transport in porous media: Multirate interaction by exchange with the mean. Water Resources Research 56(8):e2019WR026,993, doi:10.1029/2019WR026993

Souzy M, Lhuissier H, Méheust Y, Le Borgne T, Metzger B (2020) Velocity distributions, dispersion and stretching in three-dimensional porous media. Journal of Fluid Mechanics 891:A16, doi:10.1017/jfm.2020.113

Suciu N, Radu F, Attinger S, Schüler L, Knabner P (2015) A fokker-planck approach for probability distributions of species concentrations transported in heterogeneous media. Journal of Computational and Applied Mathematics 289:241-252, doi:10.1016/j.cam.2015.01.030

Tolbin AY, Pushkarev VE, Tomilova LG (2018) A mathematical analysis of deviations from linearity of beer's law. Chemical Physics Letters 706:520 - 524, doi:10.1016/j.cplett.2018.06.056

Valocchi AJ, Bolster D, Werth CJ (2019) Mixing-limited reactions in porous media. Transport in Porous Media 130(1):157-182, doi:10.1007/s11242-018-1204-1

Zou R, Yu A (1995) The packing of spheres in a cylindrical container: the thickness effect. Chemical Engineering Science 50(9):1504-1507, doi:10.1016/0009-2509(94)00483-8

\section{Appendix: Estimating pore size distributions}

To estimate the distributions of inscribed pore diameters we use an arbitrary cubic sub-sample of each medium, with dimensions $\left(9 d_{0}\right)^{3}$. We first discretize the space in equal cubic voxels of size $d_{0} / 60$ and compute, for each fluid phase voxel, its associated minimum Eulerian distance to any solid phase voxel. The map containing such values is usually referred to as Eulerian Distance transform or EDT. We then follow the morphology analysis principles of Silin and Patzek (2006), who define an individual pore as being composed of a master voxel (which holds the local maximum EDT value) and a set of connected slave voxels. A voxel with associated value $\mathrm{EDT}_{\mathrm{S}}$ is a slave of another voxel with $\mathrm{EDT}_{\mathrm{M}}$ if: $(i) \mathrm{EDT}_{\mathrm{M}} \geq \mathrm{EDT}_{\mathrm{S}}$ and $(i i) \Delta_{\mathrm{MS}} \leq \mathrm{EDT}_{\mathrm{M}}+\mathrm{EDT}_{\mathrm{S}}$, where $\Delta_{\mathrm{MS}}$ is their mutual distance. A hierarchy can then be defined such that "my slave's slave is also my slave". The voxels that remain "unslaved" are the pores' master voxels, and their respective EDT $_{M}$ values correspond to each pore's maximum inscribed radius. Finally, the empirical distributions are fit to a generalized extreme value model, which agrees very well with the empirical data (see Figure 2(b)) showing the best indicators, for both the Bayesian and Akaike information criteria, among a wide variety of tested distributions. The empirically determined pore size distribution in the mono-disperse case appears to correctly reflect the geometrical limit $\phi / d_{0} \geq 0.22$, which corresponds to a close tetrahedral stack of four equal spheres. The comparison between Figure 2(a) and Figure 2(b) demonstrates that the three log-normal grain-size distributions, built with different mean and variance to have matching hydraulic radius, yield pore-size distributions with equal mode and a smaller difference in variance. In other words, these results suggest a close relationship between the mean hydraulic radius and the typical pore size, for granular media of the kind considered here. 


\section{Supplementary Files}

This is a list of supplementary files associated with this preprint. Click to download.

- ACloserLook.pdf 